\title{
Comparative Monte Carlo Study of a Monolayer Growth in a Heteroepitaxial System in the Presence of Surface Defects
}

\author{
M. Cecilia Giménez, Ezequiel P. M. Leiva * \\ Unidad de Matemática y Física, Facultad de Ciencias Químicas \\ INFIQC. \\ Universidad Nacional de Córdoba, 5000 \\ Córdoba, Argentina
}

September 12, 2021

\begin{abstract}
The adsorption of a metal monolayer or submonolayer for the systems $\mathrm{Ag} / \mathrm{Au}(100), \mathrm{Au} / \mathrm{Ag}(100), \mathrm{Ag} / \mathrm{Pt}(100), \mathrm{Pt} / \mathrm{Ag}(100), \mathrm{Au} / \mathrm{Pt}(100)$, $\mathrm{Pt} / \mathrm{Au}(100), \mathrm{Au} / \mathrm{Pd}(100)$ and $\mathrm{Pd} / \mathrm{Au}(100)$ was studied by means of lattice Monte Carlo simulations.

It was found that, taking into account some general trends, such systems can be classified into two big groups. The first one comprises $\mathrm{Ag} / \mathrm{Au}(100), \mathrm{Ag} / \mathrm{Pt}(100), \mathrm{Au} / \mathrm{Pt}(100)$ and $\mathrm{Au} / \mathrm{Pd}(100)$, which have favourable binding energies as compared with the homoepitaxial growth of adsorbate-type atoms. When the simulations are performed in the presence of substrate-type island in order to emulate surface defects, the islands remain almost unchanged, and the adsorbate atoms successively occupy kink sites, step sites and the complete monolayer.

The second group is composed of $\mathrm{Au} / \mathrm{Ag}(100), \mathrm{Pt} / \mathrm{Ag}(100), \mathrm{Pt} / \mathrm{Au}(100)$, and $\mathrm{Pd} / \mathrm{Au}(100)$, for which monolayer adsorption is more favourable on substrates of the same nature than on the considered substrates. When simulations are carried out in the presence of islands of substratetype atoms, these tend to disintegrate in order to form 2-D alloys with adsorbate atoms.

Keywords: metal deposition, embedded atom method, Monte Carlo simulations
\end{abstract}

\footnotetext{
${ }^{*}$ Corresponding author. Fax 54-351-4344972; e-mail: eleiva@fcq.unc.edu.ar
} 


\section{Introduction}

Research on the electrochemical deposition of a metal $M$ onto a well-ordered single crystalline surface of a foreign metal $S$ should provide a better understanding of the fundamental aspects of metal deposition [1-8]. When this occurs at potentials more positive than those predicted from the Nernst equation, the process is denominated underpotential deposition(upd) [2, 3, 4, 5], to differenciate it from the usual deposition process at overpotentials. Due to the high complexity of the deposition phenomenon, no general theory has been able to embody the behavior of metal monolayers and submonolayers formed on a foreign single-crystal surface. This is partly due to the fact that, even in this simple case, the process is complicated by the appearance of several phases, involving expanded structures, anion adsorption, formation of surface alloys, etc. While each system may require its own specific modelling in order for its fine features to be properly described, we think that comparative computational studies of some simple model systems may yield important information leading to understanding of the more complicated experimental systems. Thus, rather than seeking "experimental agreement", it may be relevant to analyze a model that contains some key features that play an important role in the experiments.

It is the purpose of this work to analyze the growth of a metal on a foreign substrate in the presence of surface defects that will be represented by islands of the same material as that of the substrate. This is done in order to find trends or regularities for a number of systems involving metals of common use in electrochemistry. As they provide a realistic interaction of the metallic binding, EAM potentials [9] are employed to describe the atomatom interaction. Using the Monte Carlo method within a lattice model, allows us to deal with systems having a reasonably large number of particles.

Although the model chosen may seem simple as compared with reality, the rich behavior of the present simulations provides several clues to understand the behavior of some experimental systems, and suggests a number of future studies that may be tackled by experimentalists in the area. The present comparative study involves the systems $\mathrm{Ag} / \mathrm{Au}(100), \mathrm{Au} / \mathrm{Ag}(100), \mathrm{Ag} / \mathrm{Pt}(100)$, $\mathrm{Pt} / \mathrm{Ag}(100), \mathrm{Au} / \mathrm{Pt}(100)$ and $\mathrm{Pt} / \mathrm{Au}(100), \mathrm{Pd} / \mathrm{Au}(100)$ and $\mathrm{Au} / \mathrm{Pd}(100)$. 


\section{$2 \quad$ Model and simulation method}

\section{$2.1 \quad$ Lattice model}

As they allow to deal with a large number of particles at a relatively low computational cost, lattice models are widely used in computer simulations to study nucleation and growth. In principle, it must be kept in mind that continuum Hamiltonians, where particles are allowed to take any position in space, are much more realistic in cases where epitaxial growth of an adsorbate leads to incommensurate adsorbed phases [10] or to adsorbates with large coincidence cells. On the other hand, to assume that particle adsorption can only occur at definite sites is a good approximation for some systems. Such is the case of silver on gold, where there is no crystallographic misfit. We have studied this system before [11] and now we apply the same assumption to other systems in order to perform a comparative analysis when adsorption takes place in the presence of surface defects.

With this purpose, we employ here a lattice model to represent the square (100) surface lattice in a Grand Canonical Monte Carlo simulation. Besides adsorbate adatoms, atoms of the same nature as the substrate are present in the monolayer at a coverage degree of 0.1 , with different surface structures so as to emulate some of the most common surface defects. In order to obtain the adsorption isotherms and study the influence of the surface defects on their shapes, we calculate the average coverage degree at each chemical potential. Solvent effects are neglected, but this should not be a major problem for the metal couples considered, since the partial charge on the adatoms is expected to be small, thus minimizing the dipole-dipole interactions. We also neglect all kinds of anion effects that may coadsorb during the metal deposition process. This may lead to some underestimation or overestimation of the underpotential shift defined below [2] depending on wether anions adsorb more strongly or more weakly on the adsorbate than on the substrate[12.

Square lattices of size $(100 \times 100)$ with periodical boundary conditions are used here to represent the surface. Each lattice node represents an adsorption site for an atom. The adsorbate may adsorb, desorb or hop between neighboring sites, while the atoms of the same nature as the substrate may only move on the surface like the other atoms do. In this way, our model corresponds to an open system for one of its components, as it is the case of adatom deposition on a foreign surface.

Different structures can be chosen as initial conditions for each simulation. In the present case, we start with islands of atoms of the same nature as the substrate of different sizes and shapes obtained by means of simulated annealing techniques. This was undertaken in order to imitate some 
of the defects that can be found on a real single crystal surface, like kink sites, isolated substrate atoms, steps, etc. At each chemical potential, the initial condition is the monolayer with surface defects generated by simulatted annealing of 1000 substrate atoms, as will be discussed later, and 2000 adsorbate atoms, giving an initial coverage degree of $\theta=0.222$.

\subsection{Energy Calculation}

To calculate the activation energies for adatom diffusion the Embedded Atom Method (EAM) was used [9]. This method takes into account many-body effects; therefore, it represents the metallic bonding better than a pair potential does. The total energy of the system is calculated as the sum of the energies of the individual particles. Each energy is in turn the sum of an embedding (attractive) energy and a repulsive contribution which arises from the interaction between ion cores. The EAM contains parameters which were fitted to reproduce experimental data, such as elastic constants, enthalpies of binary alloys dissolution, lattice constants, and sublimation heats.

The application of the EAM within a lattice model is described in detail in our previous work [11]. In the present work some numerical results may differ from those obtained in the previous one due to the fact that we had used 'single zeta' functions and now we use 'double zeta' functions for the calculation of the energies [13, 14]. Nevertheless, in both works the qualitative results and the conclusions are the same.

\subsection{Grand Canonical Monte Carlo}

One of the most appealing characteristics of Grand Canonical Monte Carlo $(\mu V T / M C)$ is that, like in many experimental situations, the chemical potential $\mu$ is one of the independent variables. This is the case of low-sweep rate voltammetry, an electrochemical technique where the electrode potential can be used to control the chemical potential of species at the metal/solution interface. This technique offers a straightforward way of obtaining the adsorption isotherms provided the sweep rate is low enough to ensure equilibrium for the particular system considered. In the solid-vacuum interface, the chemical potential is related to the vapor pressure of the gas in equilibrium with the surface. However, chemical potential sweeps under equilibrium conditions cannot be achieved in the solid-vacuum interface because the desorption process is too slow due to the high energy barrier that the metal adatoms have to surmount (typically of the order of some eVs).

Our 2D system is characterized by a square lattice with $M$ adsorption sites. We labelled each adsorption site 0,1 or 2 , depending on whether it is 
empty, occupied by one substrate type atom or occupied by one adsorbate atom respectively.

Following the procedure proposed by Metropolis and coworkers [15, the acceptance probability for a transition from state $\vec{n}$ to $\vec{n}^{\prime}$ is defined as:

$$
W_{\vec{n} \rightarrow \vec{n}^{\prime}}=\min \left(1, \frac{P_{\vec{n}^{\prime}}}{P_{\vec{n}}}\right)
$$

so that detailed balance is granted.

In our $\mu V T$ / $M C$ simulation we shall allow for three types of events:

1. Adsorption of an adsorbate atom onto a randomly-selected lattice site.

2. Desorption of an adsorbate atom from an occupied lattice site selected at random.

3. Motion of an atom from the lattice site where it is adsorbed to one of its four nearest neighbor sites. The latter is selected randomly.

Even when in a grand canonical simulation, events of type 3 (motion or diffusion of lattice particles) are not strictly necessary, their presence is justified by the fact that a smaller number of Monte Carlo steps(MCS) must be employed for the equilibration of the system [15]. It is also important in the case of motion of substrate type atoms, which would not be possible without that type of events.

Within this procedure, the relevant thermodynamic properties are then obtained, after some equilibration steps, as average values of instantaneous magnitudes stored along a simulation run. In the present case, we are interested in the average coverage degree of the adsorbate atoms $\langle\Theta\rangle_{\text {Ads }}$ at a given chemical potential $\mu$, where the instantaneous value, $\Theta(\mu)_{A d s, i}$, is defined as follows:

$$
\Theta(\mu)_{A d s, i}=\frac{N_{A d s, i}}{M-N_{S u}}
$$

where $N_{A d s, i}$ is the number of adsorbate atoms, $M$ is the total number of sites and $N_{S u}$ is the number of substrate-type atoms present on the surface at the time step $i$.

Since our main goal is to obtain adsorption isotherms for different surface structures of substrate atoms, we choose a simulation method which does not yield information on the kinetics of nucleation and growth. The Kinetic Monte Carlo (KMC) [16, 17] technique could be applied with this purpose. Studies in this direction are under way [18, 19]. 


\subsection{Algorithm employed for the calculation of energy differences}

One of the main advantages of the lattice model is its simplicity, since it fixes the distances between the adsorption nodes, thus reducing the energy values that the system can take to a discrete set. Furthermore, the potentials used are short ranged, so that a very important simplifying assumption can be made for obtaining $\Delta U$. The point is to consider the adsorption(desorption) of a particle at a node immersed in a certain environment, as shown in Figure 1 . The adsorption site for the particle is located in the central box, and the calculation of the interactions is limited to a circle of radius $R$. Then, the adsorption energy for all the possible configurations of the environment of the central atom can be calculated previous to the simulation. In this case, we employ configurations which include first, second and third nearest neighbors, given a total of 13 sites, including the central atom (under consideration). With this method all the adsorption energies of an atom are tabulated, so that during the MC simulation the most expensive numerical operations are reduced to the reconstruction of the number $I$ that characterizes the configuration surrounding the particle on the adsorption node. Computationally speaking, $I$ is nothing but the index of the array in which the energy is stored.

In all the structures generated, the plane of the adsorbates was located at a vertical distance from the first substrate plane equivalent to the distance between the substrate and one complete adsorbate monolayer. This value was previously obtained by minimizing the energy as a function of that distance.

\subsection{Simulated annealing}

Simulated annealing techniques have often been used to obtain minimal energy structures or to solve ergodicity problems. A suitable way to implement them is through the canonical Monte Carlo method at different temperatures. In the present case, we used simulated annealing in order to obtain different surface defects given by islands of various sizes and shapes. In all cases the initial state involved a coverage degree of 0.1 substrate atoms distributed at random.

The simulation was started at a very high initial temperature $T_{o}$, of the order of $10^{4} \mathrm{~K}$, and the system was later cooled down following a logarithmic law:

$$
T_{f}=T_{o} K^{N_{\text {cycles }}}
$$


where $\mathrm{T}_{f}$ is the final temperature, $N_{\text {cycles }}$ is the number of cooling steps and $K$ is a positive constant lower than one (in this case, we use $K=0.9$ ). A certain number of MCS were run at each temperature and the simulation stoped when $T_{f}$ was reached. The number of MCS employed at each temperature varied from 20 to 655360 , giving different kinds of structures as we shall discuss later.

\subsection{Underpotential vs overpotential deposition}

The possibility that upd takes place can be quantified through the so-called underpotential shift $\Delta \phi_{U P D}$, which is related to the difference of the chemical potential of $M$ adsorbed on $S$ at a coverage degree $\Theta$, say $\mu\left(M_{\Theta} / S\right)$, and the chemical potential of $M$ in the bulk phase, say $\mu(M / M)$, through the

following equation:

$$
\Delta \phi_{U P D}=\frac{1}{z e_{0}}\left[\mu(M / M)-\mu\left(M_{\Theta} / S\right)\right]
$$

where $z$ is the charge of the ion $M$ in the solution and $e_{0}$ is the elemental charge. Since $\Delta \phi_{U P D}$ depends on the valence of the ion being deposited, we prefer to use the excess of chemical potential $\Delta \mu$ as stability criteria for adsorbed monolayers which we define as:

$$
\Delta \mu=\mu\left(M_{\Theta} / S\right)-\mu(M / M)
$$

According to equations (4) and (5), negative values of $\Delta \mu$ indicate underpotential deposition, while positive ones predict overpotential deposition.

\section{Results and discussion}

We have considered the following systems: $\mathrm{Ag}$ on $\mathrm{Au}(100)$; $\mathrm{Au}$ on $\mathrm{Ag}(100)$; $\mathrm{Ag}$ on $\mathrm{Pt}(100)$; Pt on $\mathrm{Ag}(100)$; $\mathrm{Au}$ on $\mathrm{Pt}(100)$; Pt on $\mathrm{Au}(100), \mathrm{Pd}$ on $\mathrm{Au}(100)$ and $\mathrm{Au}$ on $\operatorname{Pd}(100)$. In the following discussion and for the sake of simplicity we omit the Miller indices denoting the crystal surface, writing first the adsobate and then the substrate, i.e. instead of $\mathrm{Ag}$ on $\mathrm{Au}(100)$ we write $\mathrm{Ag} / \mathrm{Au}$. 


\subsection{Energy tables}

In figure 1 we show 25 relevant configurations out of the 531441 which are possible for the environments that an atom can find on the surface. The corresponding adsorption energies are summarized as illustrative examples in table I. Configurations 1-17 and 24 correspond to environments involving only adsorbate atoms. Comparing configurations 1, 2, 3, 4 and 5, we can see the influence of the first neighbors, which in all cases have a favorable effect on the adsorption of the central atom. By comparing cases $6,7,8$, 9 and 10, we can see the influence of second neighbors(in the absence of first neighbors) and we can conclude that, except for $\mathrm{Ag} / \mathrm{Pt}$, they favor in all cases the adsorption of an atom at the central site. Nevertheless, the influence of second neighbors seems to be the opposite when first neighbors are present (see configurations 11 and 13 as compared with configuration 2). This is due to the non-linear effects of the many body interaction energies: the binding of the second neighbor to the first one weakens the binding of the central atom to the first neighbor. The direct influence of third neighbors is almost negligible (compare configurations 1 and 16). On the other hand, the presence of a third neighbor close to a first neighbor tends to be unfavorable for adsorption of the central atom, for the same reasons stated in the previous case (compare configurations 2 and 17).

Configurations 19 and 20 are meaningful when considering adsorption at step sites. In this respect, observation of Table I indicates that when adsorbing on $\mathrm{Pt}$ steps $\mathrm{Ag}$ and $\mathrm{Au}$ atoms will avoid neighboring homoatoms. This is not unexpected, since both $\mathrm{Ag}$ and $\mathrm{Au}$ exhibit an important compressive surface stress when adsorbed on Pt. On the other hand, the adatoms of the other systems will prefer to adsorb beside other adatoms.

Configurations 21, 22 and 23 represent the environment of the three kinds of kink sites that an atom can find on the surface. Although no conclusion can be drawn about a general trend, it can be stated that the energetic behavior is monotonic, in the sense that the strength of the binding increases or decreases with the coordination of the kink site for the three types of sites.

Comparing configurations 20 and 22 for all systems, it can be noticed that for the systems $\mathrm{Ag} / \mathrm{Au}, \mathrm{Ag} / \mathrm{Pt}$ and $\mathrm{Au} / \mathrm{Pt}$, adsorption onto kink sites(conf. 22 ) is favored as compared with step decoration (conf. 20), whereas for the systems $\mathrm{Au} / \mathrm{Ag}, \mathrm{Pt} / \mathrm{Ag}, \mathrm{Pt} / \mathrm{Au}, \mathrm{Au} / \mathrm{Pd}$ and $\mathrm{Pd} / \mathrm{Au}$ the growing of a monodimentional phase at step sites should be preferred (conf. 20). This has important consequences for the sequential filling of the different types of deffects. In the case of the former family of systems, kink sites should be filled before step decoration occurs, so that in the adsorption isotherm the two processes should appear sequentially. On the other hand, for the second 
type of systems a sequential behavior should not be expected. We shall return later to this phenomenon when analyzing the adsorption isotherms. A similar trend can be noticed when comparing configurations 14 and 19, which correspond to the adsorption of an adatom close to a step of the same (conf. 14) or a different (conf. 19) nature. For the systems $\mathrm{Ag} / \mathrm{Au}$, $\mathrm{Ag} / \mathrm{Pt}, \mathrm{Au} / \mathrm{Pt}$ and $\mathrm{Au} / \mathrm{Pd}$, configuration 19 is more stable than conf. 14, so that in these cases, the adsorption of a new atom at the step of a substrate island should be more favourable than adsorption on the edge of an adsorbate island. The opposite trend is observed for the systems $\mathrm{Au} / \mathrm{Ag}, \mathrm{Pt} / \mathrm{Ag}, \mathrm{Pt} / \mathrm{Au}$ and $\mathrm{Pd} / \mathrm{Au}$. However the system $\mathrm{Pd} / \mathrm{Au}$ is a borderline case, as the energy difference between configurations 14 and 19 is of the order of $0.03 \mathrm{eV}$, that is, close to $\mathrm{kT}$ at room temperature.

\subsection{Adsorption isotherms in defect free surfaces}

Adsorption isotherms were calculated for the different systems in the case of defect free surfaces. For a fixed temperature and chemical potential a simulation was performed and the average coverage degree was calculated for the equilibrium state of the system. As this was repeated at different chemical potentials, the isotherms obtained were like those represented in figure 2 for the case of the systems $\mathrm{Ag} / \mathrm{Au}$ and $\mathrm{Au} / \mathrm{Ag}$. The isotherms were performed at a temperature of $300 \mathrm{~K}$ and we include two in each graphic: $\mathrm{A} / \mathrm{S}(100)$ and $\mathrm{A} / \mathrm{A}(100)$, where $\mathrm{A}$ and $\mathrm{S}$ denote adsorbate and substrate respectively. Thus comparison of the behavior of the heteroatomic A/S(100) system with that of the pure metal A/A(100) system allows to determine the existence of underpotential or overpotential deposition. As we can see, for each isotherm there is an abrupt jump in the coverage degree as expected in the case of a first order phase transition. As we discussed in a previous work [11, at higher temperatures the isotherms tend to become smoother, approximating to the shape of a Langmuir isotherm.

According to equations (44) and (5), the difference between the chemical potentials $\Delta \mu$ at which transition occurs for the systems $\mathrm{A} / \mathrm{S}(100)$ and $\mathrm{A} / \mathrm{A}(100)$ is a measure of the underpotential shift:

$$
\Delta \phi_{U P D}=-\frac{\Delta \mu}{z e_{0}}
$$

In table 2 , we summarize the approximate values for the chemical potential at which transition occurs for each system. In table 3 we show the excess of chemical potential for the systems A/S(100) as defined in equation (5), calculated as the difference of the values of the previous table for the systems A/S(100) and A/A(100). Negative values predict underpotential deposition, while positive ones predict overpotential deposition. These 
results agree well with those obtained in previous works [20, 21] by means of a thermodynamic cycle using the embedded atom method. According to these results, upd is predicted for the systems $\mathrm{Ag} / \mathrm{Au}, \mathrm{Ag} / \mathrm{Pt}, \mathrm{Au} / \mathrm{Pt}$ and $\mathrm{Au} / \mathrm{Pd}$, and overpotential deposition is expected for the remaining systems. Although this prediction is in agreement with the experimental results for $\mathrm{Ag} / \mathrm{Au}$ and $\mathrm{Ag} / \mathrm{Pt}$, it does not agree with experimental finding of upd for $\mathrm{Pd} / \mathrm{Au}[23]$. It is also interesting to point out that this system seems to go against the empirical rule that upd should occur when the work function of the metal being deposited is lower than that of the substrate ( $\Phi_{A u}^{111}=$ $\left.5.31 \mathrm{eV} ; \Phi_{A u}^{100}=5.47 \mathrm{eV} ; \Phi_{A u}^{110}=5.37 \mathrm{eV}, \Phi_{P d}^{111}=5.6 \mathrm{eV}[22]\right)$. Underpotential deposition for this system also goes against the intuitive expectation that it should occur when the surface energy $\sigma$ of the substrate is larger than that of the adsorbate, $\left(\sigma^{A u}=0.094 \mathrm{eV} / \AA^{2} ; \sigma^{P d}=0.125 \mathrm{eV} / \AA^{2},[9]\right.$ ). First principles calculations also yield a negative excess of binding energy[12], so that this system presents some remarkable singularities. It must be emphasized, however, that Pd deposition occurs in the presence of strongly adsorbed chloride anions which result in a distorted-hexagon cloride on top of the $\mathrm{Pd}$ monolayer 23. Thus the presence of adsorbed anions may yield part of the energy excess required for the existence of upd.

\subsection{Surface defects}

In order to study the influence of surface defects on the adsorption isotherms, simulations were performed in the presence of substrate atoms in the monolayer, with a coverage degree of 0.1 . With the present simulation parameters, such coverage degree corresponds to 1000 atoms as initial conditions. Different surface defects were generated by employing various cooling rates in the simulated annealing procedure described above. This was achieved using different numbers of Monte Carlo steps $N_{s}$ at each temperature. Five defective surface types were created, using $N_{s}=20$ for surface type $1, N_{s}=320$ for surface type $2, N_{s}=5120$ for surface type $3, N_{s}=81920$ for surface type 4 and $N_{s}=655360$ for surface type 5 . Surface type 1 contains the largest quantity of islands and they are smaller than those of other surfaces. The total number of islands decreases from surface types 1 to 5 and their size increase accordingly. This implies that the number of kink sites and the number of step sites also decrease from surface type 1 to 5 . In the following figures, these defective surface types are labelled according to the average island size $<s_{i}>$ obtained, which were for example 7, 13, 48, 200 and 500 for the system $\mathrm{Ag} / \mathrm{Au}$. 


\subsubsection{Adsorption isotherms}

As can be noticed in figure 2, adsorption isotherms on defect-free surfaces show an abrupt change of coverage degree for a certain critical chemical potential. This situation turns to be different in the presence of surface defects for the systems $\mathrm{Ag} / \mathrm{Au}, \mathrm{Ag} / \mathrm{Pt}, \mathrm{Au} / \mathrm{Pd}$ and $\mathrm{Au} / \mathrm{Pt}$ (Figure 3). In these cases the coverage degree starts to rise slowly at chemical potentials more negative than the critical chemical potential. This effect is more important in the case of surfaces with smaller islands, which contain more kink and step sites. Observation of the status of the surface, discussed in detail below, shows that these are the places that are occupied first. Relative coverage degrees $\Theta_{k}\left(\Theta_{s}\right)$ for kink (step) sites may be defined as the number of occupied kink (step) sites divided by the total number of kink (step) sites. Figures 4 and 5 show $\Theta_{k}, \Theta_{s}$, and the total coverage degree $\Theta$ as a function of $\mu_{M}$. The behavior of these isotherms indicates that kink sites are occupied first, then, step sites, and finally, the rest of the surface. Close inspection of these figures shows that the chemical potential values at which $\Theta_{k}=0.5$ are close to the corresponding energy values for adsorption at kink sites (see configurations 21-23 in figure 1 and corresponding values in Table 1). Something similar occurs with the $\mu_{M}$ values at which $\Theta_{s}=0.5$, which are close to the energy values of configuration 20 in figure 1 (see also entry 20 in Table 1). However, it must be noticed that an abrupt jump is not observed in the $\Theta_{k}$ and $\Theta_{s}$ isotherms, as expected for the 0 and 1 dimensional systems.

Unlike the systems considered in figure 3, the systems $\mathrm{Au} / \mathrm{Ag}, \mathrm{Pt} / \mathrm{Ag}$, $\mathrm{Pd} / \mathrm{Au}$ and $\mathrm{Pt} / \mathrm{Au}$ do not present an appreciable widening of the adsorption isotherms with increasing number of surface defects (Figure 6). Figures 7 and 8 show that the partial coverages $\Theta_{k}$ and $\Theta_{s}$ for this type of systems do not exhibit a clearcut trend as the systems depicted in figures 4 and 5 . This can be understood through comparative analysis of configuration 20 against configurations 21-23. It is clear that these systems do not prefer adsorption at steps to adsorption at kink sites, in contrast to the prediction for the systems presented in figure 3. Thus kink sites are not filled before step decoration, but both processes take place simultaneously. Furthermore, as pointed out above, configuration 14 yields lower energy values than configuration 19 does, so that atom deposition at substrate island steps is delayed with respect to deposition at an island of the same nature. It is also remarkable that within the present model all these systems exhibit a positive excess of binding energy, and that the binding energy of the adsorbate is larger than, or similar to that of the substrate (See table 4). 


\subsubsection{Morphology}

It is very illustrative to analyze the final state of the surface of some typical systems at different chemical potentials. In figure 9 , we show frames corresponding to the final state of the $\mathrm{Ag} / \mathrm{Pt}$ system at 6 different chemical potentials. In principle, the islands are not fixed and all atoms are allowed to diffuse, however, there appears to be no movement of the substrate type atoms, so that the islands remain unchanged. The adsorbate atoms occupy the free sites with the preferences described above, that is, first filling the kink sites, then the steps, and finally the rest of the system. A similar behavior is observed for the systems $\mathrm{Ag} / \mathrm{Au}$ and $\mathrm{Au} / \mathrm{Pt}$. The situation is strikingly different for the systems $\mathrm{Au} / \mathrm{Ag}, \mathrm{Pt} / \mathrm{Ag}, \mathrm{Pd} / \mathrm{Au}$ and $\mathrm{Pt} / \mathrm{Au}$, in all of which, adsorbates come into the islands forming an alloy and these tend to disintegrate. This is illustrated in figure 10 for Pt deposition on $\mathrm{Ag}(100)$. At small coverage degrees the adatoms start to get already embedded into the islands, and even form small nucleii. This process continues and upon completion of the monolayer the substrate islands have incorporated an important number of adatoms. This phenomenon also occurs for larger islands, as can be observed in figure 11, where this process is illustrated for Pt deposition on an Ag surface exhibiting a big 1000-atom island. In this case, and for larger coverage degrees, a higher concentration of adatoms results on the edge of the islands due to a slower equilibration of the system. The system $\mathrm{Au} / \mathrm{Pd}$ is an intermediate case.

A detailed view of the neighborhood of an island is presented in figures 12 and 13 for the systems $\mathrm{Au} / \mathrm{Pt}$ and $\mathrm{Pt} / \mathrm{Au}$, showing the features mentioned in the previous discussion.

While the simplicity of the present model (not considering surface reconstruction, anion adsorption and specific kinetic features) does not allow a quantitative comparison with experiments, qualitative predictions can be made concerning surface alloy formation in the presence of islands. In table 5 we present the predictions for the systems simulated in this work along with some results of experimental observation. In connection with the present results, it is worth mentioning the ones obtained by Kolb and coworkers for $\mathrm{Pd}$ and $\mathrm{Pt}$ deposition on $\mathrm{Au}(100)$ [23, 24]. For the former system, the authors proposed that alloying upon Pd deposition should proceed involving $\mathrm{Au}$ atoms from islands and step edges. The present results strongly support this explanation on thermodynamic grounds, based on the energetics of the $\mathrm{Pd} / \mathrm{Au}$ system. Interesting results have also been obtained for the second system, Pt/Au. Waibel et al [24] have studied Pt deposition on $\mathrm{Au}(100)$, finding that nucleation of Pt starts mainly at deffects like step edges for low deposition rates. On the other hand, at high deposition rates some nucleii 
also appear on the terraces at random sites. Figure 7 of reference [24] shows that over $\mathrm{Pt}$ deposition the island shape becomes progressively blurred as $\mathrm{Pt}$ is deposited, and it is very stable. According to the present results, at low deposition rates $\mathrm{Pt}$ atoms could be incorporating into the islands, yielding the enhanced stability observed. Due to its high binding energy, $\mathrm{Pt}$ is expected to present 3-D growth, as pointed out by Waibel et al, but this feature is not considered in the present model.

\subsubsection{First neighbor site occupation}

In order to get a more complete picture of phase growth in the presence of substrate islands, the average number of first neighbors of a given atom type $(M$ or $S$ ) surrounding an occupied site (with $M$ or $S$ ) was also analyzed. $n_{A}^{B}$ will denote the average number of neighbors of $B$ type atoms surrounding an $A$ type atom. Let us consider first the system $\mathrm{Au} / \mathrm{Pt}$, depicted on the left side in the lower part of figure 4. It can be seen that the average number of Pt atoms surrounding other Pt atoms as nearest neighbors, $n_{P t}^{P t}$, remains constant, at a value somewhat lower than four. This shows that the coordination of $\mathrm{Pt}$ atoms on the islands does not change upon $\mathrm{Au}$ deposition onto $\mathrm{Pt}$, in agreement with the fact that $\mathrm{Pt}$ atoms remain almost immobile. As expected, simulations with larger Pt islands yield $n_{P t}^{P t}$ closer to 4 , while the reverse occurs with simulations with smaller islands. The number of $\mathrm{Au}$ atoms around $\mathrm{Pt}, n_{P t}^{A u}$, rises from zero at chemical potentials where $A u$ is absent, to a number less than one, when the monolayer is complete. This is due to the fact that only $\mathrm{Pt}$ atoms on the edge of the islands are in contact with adsorbate atoms. Two steps can be noticed in the $n_{P t}^{A u}-\mu$ curve. The first one occurs when the kink sites of the Pt islands become occupied by $\mathrm{Au}$ atoms, and the second one when the edges of the islands (step sites) become decorated with Au. By observing the average number of $P t$ atoms around $A u, n_{A u}^{P t}$,it can be noticed that $n_{A u}^{P t} \approx 2$ in regions where only kink sites are covered, close to one in regions where step sites are covered, and very small upon completion of the $A u$ monolayer. Some isolated cases with $n_{A u}^{P t}=3$ can be observed for low coverage degrees. This is due to the fact that individual atoms of adsorbate manage to incorporate to the edge of the island, but are unable to penetrate further. The number of $A u$ atoms around $A u$ atoms, $n_{A u}^{A u}$, is zero for $\Theta_{A u} \longrightarrow 0$ and in regions where only kink sites are occupied. $n_{A u}^{A u}$ then rises to a number between one and two in regions where steps sites are occupied and becomes close to four for monolayer completion. The general features of the system $\mathrm{Ag} / \mathrm{Pt}$, depicted at the bottom right part of figure 4 are similar. This picture of sequential filling of kink and step sites is also reflected by the partial isotherms at the top of figure 4: the coverage 
degree of steps sites starts to rise only when $\Theta_{k} \approx 1$.

Figure 5 shows the same analysis for the systems $\mathrm{Au} / \mathrm{Pd}$ and $\mathrm{Ag} / \mathrm{Au}$. In the lower part of the right hand side, it can be observed for the system $\mathrm{Ag} / \mathrm{Au}$ that the general trends are similar to those of the two previous systems, but the curves $n_{\text {adsorbate }}^{\text {substrate }}\left(n_{A g}^{A u}\right)$ do not present two clearly differentiated steps as before. That is, the regions with occupation of kinks and steps are not clearly diferentiated. The same can be stated for the system $\mathrm{Au} / \mathrm{Pd}$ (left hand side in figure 5). In this figure, the curve $n_{A u}^{P d}$ presents an even more remarkable behavior. At low coverage degrees, the average number of Pd atoms around $\mathrm{Au}$ is close to four, indicating that they came inside the existing substrate islands and are completely surrounded by Pd atoms. Thus, although $n_{P d}^{P d}$ remains fairly constant denoting an important island stability, some small degree of alloying occurs. Thus the $\mathrm{Au} / \mathrm{Pd}$ system is a borderline case, where some alloying may occur but islands still remain stable. The non sequential filling of kink and step sites can also be inferred from the partial isotherms in the upper part of figure 5, where it can be observed that $\Theta_{s}$ starts to rise when the decoration of kink sites is still incomplete.

The lower part of figures 7 and 8 show $n_{A}^{B}$ vs $\mu$ plots for $\mathrm{Pd} / \mathrm{Au}, \mathrm{Pt} / \mathrm{Ag}$, $\mathrm{Pt} / \mathrm{Au}$ and $\mathrm{Au} / \mathrm{Ag}$. It is evident that in all cases the number of substrate atoms around substrate atoms $\left(n_{A u}^{A u} \mathrm{y} n_{A g}^{A g}\right)$ decreases for increasing coverage degree, denoting that the islands do not remain unaltered in the presence of the adsorbate. In the case of $\mathrm{Pd}$ adsorption on $\mathrm{Au}(100)$ and $\mathrm{Au}$ adsorption on $\mathrm{Ag}(100)$, it can be noticed that at low coverage degrees the adsorbate atoms are completely embedded in the islands $\left(n_{P d}^{A u}=4\right.$ for $\mathrm{Pd} / \mathrm{Au}$ and $n_{A u}^{A g}=4$ for $\mathrm{Au} / \mathrm{Ag}$ ). This effects somehow occur for $\mathrm{Pt} / \mathrm{Ag}$ and $\mathrm{Pt} / \mathrm{Au}$, but to a lesser extent. In the case of $\mathrm{Pt}$ adsorption, the number of substrate atoms around $\mathrm{Pt}$ is smaller than four because clustering of $\mathrm{Pt}$ atoms occur within the substrate islands, even at low Pt coverage degrees. This can be observed in the frames 2-4 of figure 10 .

The behavior of $n_{\text {substrate }}^{\text {substrate }}$ at high $\mu$ for the systems $\mathrm{Pt} / \mathrm{Ag}, \mathrm{Pt} / \mathrm{Au}$ and $\mathrm{Au} / \mathrm{Ag}$ presents one very interesting feature: $n_{\text {substrate }}^{\text {substrate }}$ remains approximately constant over a certain $\mu$ region, when surface coverage by the adsorbate is close to completion. This can be observed, for instance, at the bottom of the right hand of figure 8 , where $n_{A g}^{A g}$ remains nearly constant above $\mu=-3.9 \mathrm{eV}$. As a counterpart, $n_{A g}^{A u}$ also remains constant in this region. This means that the surface presents patches of constant composition that remain stable. In other words, in those places where the islands were originally located, patches appear which exhibit a surface larger than that of the original island. This analysis complements the discussion in the last part of the previous section. 


\section{Conclusions}

1. The systems $\mathrm{Ag} / \mathrm{Au}(100), \mathrm{Ag} / \mathrm{Pt}(100), \mathrm{Au} / \mathrm{Pt}(100)$ and $\mathrm{Au} / \mathrm{Pd}(100)$ present a positive excess of binding energies (negative excess of chemical potential) as compared with the homoepitaxial growth of adsorbate type atoms, indicating that in these systems underpotential deposition is expected.

2. On the other hand, for the $\mathrm{Au} / \mathrm{Ag}(100), \mathrm{Pt} / \mathrm{Ag}(100), \mathrm{Pt} / \mathrm{Au}(100)$, and $\mathrm{Pd} / \mathrm{Au}(100)$ systems, the monolayer adsorption is more favorable on substrates of the same nature than on the substrates considered.

3. In order to emulate surface defects, simulations were also performed in the presence of surface islands made of the same metal as the substrate. For the family of systems mentioned in item 1, the islands remained almost unchanged, being decorated by the adatoms before completion of the monolayer. In the case of the systems $\mathrm{Au} / \mathrm{Pt}(100)$ and $\mathrm{Ag} / \mathrm{Pt}(100)$ the adsorbate atoms filled in a clear sequence first the kink and then the step sites. These processes are somewhat closer in the case of $\mathrm{Ag} / \mathrm{Au}(100)$ and very close in $\mathrm{Au} / \mathrm{Pd}(100)$. For the family of systems considered in item 2, the substrate islands showed disgregation in order to form 2-D alloys with the adsorbate atoms and there is no differentiation in the filling of kink, step or terrace sites.

4. The system $\mathrm{Au} / \mathrm{Pd}(100)$ presents a borderline behavior, as a small quantity of $\mathrm{Au}$ is embedded into the $\mathrm{Pd}$ islands without altering their structure. Thus it could be suggested that, except for the systems containig $\mathrm{Au}$ and $\mathrm{Pd}$, the stability of the substrate islands upon deposition of a foreing metal is mainly determined by the difference of the cohesive energies. However, this is a matter that requires further study to make a more conclusive statement.

\section{Acknowledgements}

We thank CONICET, SeCyt-UNC, Agencia Córdoba Ciencia, Program BID 1201/OC-AR PICT $N^{o}$ 06-04505.

We thank Mario Del Popolo for stimulating discussion and ideas.

Language assistance by Karina Plasencia is gratefully acknowledged. 


\section{References}

[1] D.M. Kolb, in: H. Gerischer, C.W. Tobias(Eds.). Advances in Electrochemistry and Electrochemical Engineering. vol 11, Wiley, New York 1978, page 125 .

[2] D.M. Kolb, M. Przasnyski and H. Gerischer, J. Electroanal. Chem. 1974, $54,25$.

[3] S. Trasatti, Zeits. für Phys. Chem. NF 1975, 98, 75.

[4] L. Blum, D. A. Huckaby and M. Legault, Electrochim. Acta 1996, 41, 2201.

[5] K. Jutttner, G. Staikov, W.J. Lorenz, E. Schmidt, J. Electroanal. Chem. $\mathbf{1 9 7 7}, 80,67$.

[6] E. P. M. Leiva, in "Current Topics in Electrochemistry", Council of Scientific Information, Trivandrum, India 1993, 2, 269.

[7] E. P. M. Leiva, Electrochim. Acta 1996, 41, 2185.

[8] E. Budevski, G. Staikov and W. J. Lorenz, Electrochemical Phase Formation and Growth, VCH Weinheim 1996.

[9] S. M. Foiles, M. I. Baskes and M. S. Daw, Phys. Rev. B 1986, 33,7983.

[10] M. G. Samant, M. F. Toney, G. L. Borges, L. Blum and O. R. Melroy, J. Phys. Chem 1998, 92, 220.

[11] M.C. Giménez, M.G. Del Pópolo and E.P.M. Leiva, Electrochimica Acta 1999, 45,699.

[12] C. G. Sánchez, E.P.M. Leiva and J. Kohanoff, Langmuir, C. Sánchez, E.P.M. Leiva and J. Kohanoff, Langmuir 2001, 17, 2219.

[13] E. Clementi, C. Roetti, At. Data Nucl. Data Tables 1974, 14, 177.

[14] A. D. McLean, R. S. McLean, At. Data Nucl. Data Tables 1981, 26, 197.

[15] M. P. Allen and D. J. Tildesley, Computer Simulation of Liquids, Oxford University Press 1987.

[16] G. Brown, P. A. Rickvold, M. A. Novotny and A. Wieckowski, Colloid and Surfaces A, in Press, or cond-mat/9703209. 
[17] P. Ruggerone, C. Ratsch and M. Scheffler, Growth of Ultrathin Epitaxial Layers, Vol. 8, Eds. D. A. King and D. P. Woodruff, Elsevier Science, Amsterdam 1997.

[18] M.C. Giménez, M.G. Del Pópolo, E.P.M. Leiva, S.G. García, D.R. Salinas C.E. Mayer and W.J. Lorenz, J. Electrochem. Soc. 2002, 149, E109.

[19] M.C. Giménez, M.G. Del Pópolo, E.P.M. Leiva, Langmuir 2002, 18, 9087-9094.

[20] C. G. Sánchez, M. G. Del Pópolo and E.P.M. Leiva, Surface Science 421, 59 (1999).

[21] M. I. Rojas, C. G. Sánchez, M. G. Del Pópolo and E.P.M. Leiva, Surface Science 2000, 453, 225.

[22] CRC Handbook of Chemistry and Physics, $80^{\text {th }}$ Edition, D.R. Lide, Ed., Boca Ratón 2000.

[23] L.A. Kibler, M. Kleinert and D.M. Kolb, Surface Science 2000, 461, 155.

[24] H.-F. Waibel, M. Kleinert, L.A. Kibler and D.M. Kolb, Electrochim. Acta 2002, 47, 1461. 


\section{Tables}

Table 1: Energy differences (in eV) associated with the deposition of an atom in the environments represented in figure 3 for the systems considered in this work.

\begin{tabular}{|l|l|l|l|l|l|l|l|l|}
\hline conf. & $\mathrm{Ag} / \mathrm{Au}$ & $\mathrm{Au} / \mathrm{Ag}$ & $\mathrm{Ag} / \mathrm{Pt}$ & $\mathrm{Pt} / \mathrm{Ag}$ & $\mathrm{Au} / \mathrm{Pt}$ & $\mathrm{Pt} / \mathrm{Au}$ & $\mathrm{Au} / \mathrm{Pd}$ & $\mathrm{Pd} / \mathrm{Au}$ \\
\hline 1 & -2.580 & -3.106 & -3.127 & -4.224 & -3.672 & -4.228 & -3.283 & -3.117 \\
\hline 2 & -2.866 & -3.566 & -3.340 & -4.828 & -4.160 & -4.847 & -3.901 & -3.502 \\
\hline 3 & -3.126 & -3.987 & -3.521 & -5.397 & -4.585 & -5.434 & -4.444 & -3.862 \\
\hline 4 & -3.366 & -4.380 & -3.681 & -5.938 & -4.965 & -5.986 & -4.453 & -3.863 \\
\hline 5 & -3.591 & -4.744 & -3.825 & -6.455 & -5.300 & -6.508 & -5.358 & -4.535 \\
\hline 6 & -2.582 & -3.142 & -3.122 & -4.267 & -3.705 & -4.246 & -3.335 & -3.137 \\
\hline 7 & -2.585 & -3.177 & -3.116 & -4.310 & -3.738 & -4.254 & -3.388 & -3.157 \\
\hline 8 & -2.585 & -3.177 & -3.116 & -4.310 & -3.738 & -4.264 & -3.388 & -3.157 \\
\hline 9 & -2.588 & -3.212 & -3.110 & -4.353 & -3.770 & -4.282 & -3.439 & -3.178 \\
\hline 10 & -2.590 & -3.247 & -3.105 & -4.396 & -3.802 & -4.300 & -3.490 & -3.198 \\
\hline 11 & -2.842 & -3.563 & -3.303 & -4.837 & -4.130 & -4.833 & -3.878 & -3.497 \\
\hline 12 & -2.868 & -3.599 & -3.332 & -4.869 & -4.188 & -4.863 & -3.948 & -3.521 \\
\hline 13 & -2.825 & -3.567 & -3.275 & -4.852 & -4.112 & -4.817 & -3.863 & -3.500 \\
\hline 14 & -2.810 & -3.541 & -3.259 & -4.826 & -4.071 & -4.785 & -3.815 & -3.485 \\
\hline 15 & -3.076 & -3.947 & -3.455 & -5.370 & -4.495 & -5.388 & -4.347 & -3.833 \\
\hline 16 & -2.580 & -3.106 & -3.126 & -4.224 & -3.671 & -4.227 & -3.282 & -3.116 \\
\hline 17 & -2.840 & -3.529 & -3.309 & -4.792 & -4.103 & -4.814 & -3.833 & -3.477 \\
\hline 18 & -3.002 & -3.470 & -3.692 & -4.634 & -4.336 & -4.708 & -3.905 & -3.497 \\
\hline 19 & -2.917 & -3.473 & -3.564 & -4.651 & -5.139 & -4.640 & -3.885 & -3.450 \\
\hline 20 & -3.149 & -3.859 & -3.174 & -5.185 & -4.626 & -5.193 & -4.382 & -3.777 \\
\hline 21 & -3.250 & -3.760 & -4.010 & -4.996 & -4.758 & -5.048 & -4.351 & -3.758 \\
\hline 22 & -3.230 & -3.770 & -3.976 & -5.009 & -4.747 & -5.029 & -4.363 & -3.747 \\
\hline 23 & -3.211 & -3.780 & -3.942 & -5.022 & -4.736 & -5.010 & -4.374 & -3.736 \\
\hline 24 & -3.358 & -3.498 & -3.534 & -6.257 & -4.808 & -6.176 & -4.796 & -4.384 \\
\hline 25 & -3.731 & -4.287 & -4.621 & -5.661 & -5.505 & -5.635 & -5.176 & -4.223 \\
\hline & & & & & & & &
\end{tabular}


Table 2: Chemical potential $\mu$ in $\mathrm{eV}$ at which the step is observed in the adsorption isotherms for the different systems.

\begin{tabular}{|l|l|l|l|l|}
\hline Substrate $\backslash$ Adsorbate & $A g$ & $A u$ & $P t$ & $P d$ \\
\hline$A g$ & -2.83 & -3.87 & -5.30 & -- \\
\hline$A u$ & -3.00 & -3.95 & -5.29 & -3.78 \\
\hline$P t$ & -3.38 & -4.37 & -5.83 & -- \\
\hline$P d$ & -- & -4.21 & -- & -3.92 \\
\hline
\end{tabular}

Table 3: Excess of chemical potential in $\mathrm{eV}$, as calculated from the adsorption isotherms according to equation (5).

\begin{tabular}{|l|l|l|l|l|}
\hline substrate $\backslash$ adsorbate & $A g$ & $A u$ & $P t$ & $P d$ \\
\hline$A g$ & 0.00 & 0.08 & 0.53 & - \\
\hline$A u$ & -0.17 & 0.00 & 0.54 & 0.14 \\
\hline$P t$ & -0.55 & -0.42 & 0.00 & - \\
\hline$P d$ & -- & -0.26 & - & 0.00 \\
\hline
\end{tabular}

Table 4: Cohesive energies of the bulk metal $E_{c o h}^{M(s)}[9]$.

\begin{tabular}{|l|l|l|l|l|}
\hline Metal & $\mathrm{Ag}$ & $\mathrm{Pd}$ & $\mathrm{Au}$ & $\mathrm{Pt}$ \\
\hline$E_{c o h}^{M(s)}$ & -2.85 & -3.91 & -3.93 & -5.77 \\
\hline
\end{tabular}


Table 5: Prediction of surface alloy in the presence of islands for systems simulated in this work, and experimental observations.

\begin{tabular}{|l|l|l|}
\hline System & Surface alloy prediction & Experimental observation \\
\hline $\mathrm{Ag} / \mathrm{Au}(100)$ & No & No \\
\hline $\mathrm{Ag} / \mathrm{Pt}(100)$ & No & No \\
\hline $\mathrm{Au} / \mathrm{Pd}(100)$ & Sligth alloying & Not available \\
\hline $\mathrm{Au} / \mathrm{Pt}(100)$ & No & Not Available \\
\hline $\mathrm{Au} / \mathrm{Ag}(100)$ & Yes & Nor Available \\
\hline $\mathrm{Pt} / \mathrm{Ag}(100)$ & Yes & Not Available \\
\hline $\mathrm{Pd} / \mathrm{Au}(100)$ & Yes & Surface alloying ref [23] \\
\hline $\mathrm{Pt} / \mathrm{Au}(100)$ & Yes & Au islands became stable agains dissolution ref $[24$ \\
\hline
\end{tabular}




\section{$7 \quad$ Figure Captions}

Figure 1: Some of the possible configurations associated with the adsorption of the central atom in different environments. The energies associated with all posible configurations were tabulated previous to the simulation. The energy values corresponding to these examples are shown in table 1.

Figure 2: Adsorption isotherms, plotted as coverage degree as a function of chemical potential. a) Deposition of one monolayer of Ag onto a defectfree $\mathrm{Au}(100)$ surface and one monolayer of $\mathrm{Ag}$ onto a defect-free $\mathrm{Ag}(100)$ surface. b) Deposition of one monolayer of $\mathrm{Au}$ onto a defect-free $\mathrm{Ag}(100)$ surface and one monolayer of $\mathrm{Au}$ onto a defect-free $\mathrm{Au}(100)$ surface.

Figure 3: Adsorption isotherms, plotted as coverage degree as a function of chemical potential, for the deposition of $\mathrm{Ag}$ on $\mathrm{Au}(100), \mathrm{Ag}$ on $\mathrm{Pt}(100), \mathrm{Au}$ on $\mathrm{Pd}(100)$ and $\mathrm{Au}$ on $\mathrm{Pt}(100)$ in presence of surface defects. The numbers indicate the average size of islands of substrate-type atoms present in the monolayer as initial state.

Figure 4: Adsorption isotherms for the deposition of $\mathrm{Au}$ on $\mathrm{Pt}(100)$ and $\mathrm{Ag}$ on $\mathrm{Pt}(100)$ in the presence of surface defects. Coverage degree of the monolayer, the step sites and the kink sites as a function of chemical potential (up). Average number of neighbors of each species for each kind of atom (down).

Figure 5: Adsorption isotherms for the deposition of $\mathrm{Au}$ on $\mathrm{Pd}(100)$ and $\mathrm{Ag}$ on $\mathrm{Au}(100)$ in the presence of surface defects. Coverage degree of the monolayer, the step sites and the kink sites as a function of chemical potential (up). Average number of neighbors of each species for each kind of atom (down).

Figure 6: Adsorption isotherms plotted as coverage degree as a function of chemical potential for the deposition of $\mathrm{Au}$ on a $\mathrm{Ag}(100)$, Pt on a $\mathrm{Ag}(100)$, $\mathrm{Pd}$ on $\mathrm{a} \mathrm{Au}(100)$ and $\mathrm{Pt}$ on a $\mathrm{Au}(100)$ in the presence of surface defects. The numbers indicate the average size of islands of substrate-type atoms present in the monolayer as initial state.

Figure 7: Adsorption isotherms for the deposition of $\mathrm{Pd}$ on $\mathrm{Au}(100)$ and $\mathrm{Pt}$ on $\mathrm{Ag}(100)$ in thepresence of surface defects. Coverage degree of the monolayer, the step sites and the kink sites as a function of chemical potential (up). Average number of neighbors of each species for each kind of 
atom (down).

Figure 8: Adsorption isotherms for the deposition of $\mathrm{Pt}$ on $\mathrm{Au}(100)$ and $\mathrm{Au}$ on $\mathrm{Ag}(100)$ in the presence of surface defects. Coverage degree of the monolayer, the step sites and the kink sites as a function of chemical potential (up). Average number of neighbors of each species for each kind of atom (down).

Figure 9: Snapshots of the final state of the surface at six different chemical potentials $(-4.27 \mathrm{eV},-3.97 \mathrm{eV},-3.66 \mathrm{eV},-3.44 \mathrm{eV},-3.40 \mathrm{eV}$ and -3.06 $\mathrm{eV})$ for the $\mathrm{Ag}$ on $\mathrm{Pt}(100)$ simulation. Average island size: $\langle s\rangle=48$.

Figure 10: Snapshots of the final state of the surface at six different chemical potentials $(-5.74 \mathrm{eV},-5.53 \mathrm{eV},-5.41 \mathrm{eV},-5.32 \mathrm{eV},-5.30 \mathrm{eV}$ and -5.21 $\mathrm{eV})$ for the $\mathrm{Pt}$ on $\mathrm{Ag}(100)$ simulation. Average island size: $\langle s\rangle=53$.

Figure 11: Snapshots of the final state of the surface at six different chemical potentials $(-5.74 \mathrm{eV},-5.53 \mathrm{eV},-5.41 \mathrm{eV},-5.32 \mathrm{eV},-5.30 \mathrm{eV}$ and -5.21 $\mathrm{eV})$ for the $\mathrm{Pt}$ on $\mathrm{Ag}(100)$ simulation. Average island size: $\langle s\rangle=1000$. (only one island).

Figure 12: Final state of the surface at four different chemical potentials $(-5.10 \mathrm{eV},-4.61 \mathrm{eV},-4.50 \mathrm{eV}$ and $-4.39 \mathrm{eV})$ for the $\mathrm{Au}$ on $\mathrm{Pt}(100)$ simulation with defects. One Pt island. White circles: gold atoms. Black circles: Pt atoms.

Figure 13: Final state of the surface at four diferent chemical potentials $(-5.65 \mathrm{eV},-5.30 \mathrm{eV},-5.29 \mathrm{eV}$ and $-5.26 \mathrm{eV})$ for the $\mathrm{Pt}$ on $\mathrm{Au}(100)$ simulation with defects. White circles: gold atoms. Black circles: Pt atoms. 


\section{$\underline{\mathrm{A} / \mathrm{S}(\mathbf{1 0 0})}$}

A

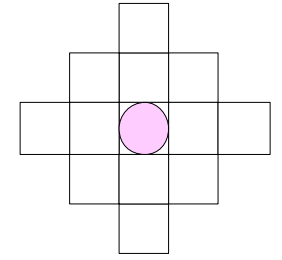

1

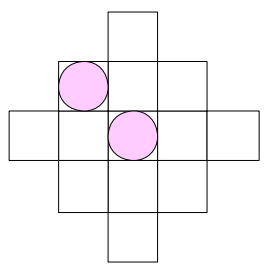

6

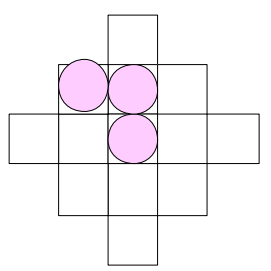

11

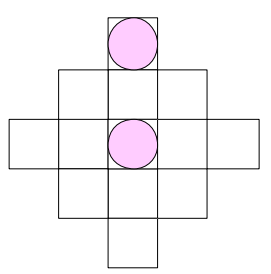

16

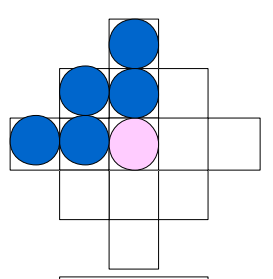

21

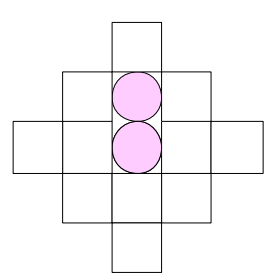

2

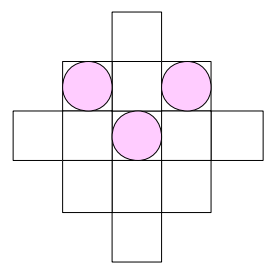

7

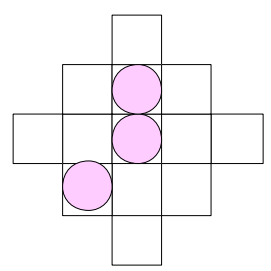

12

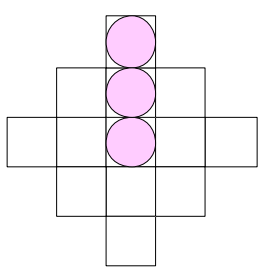

17

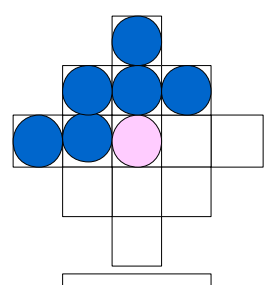

22

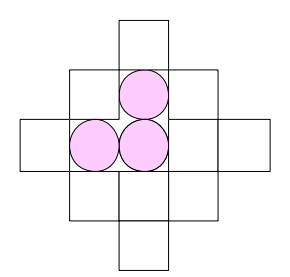

3

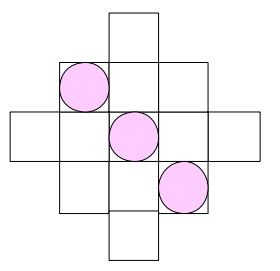

8

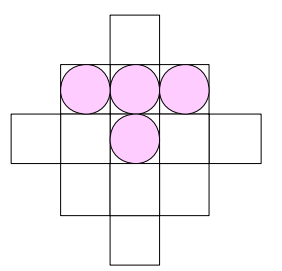

13

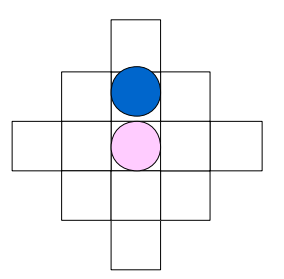

18

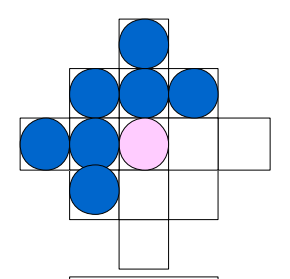

23

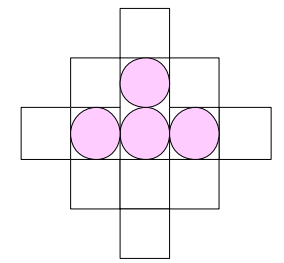

4

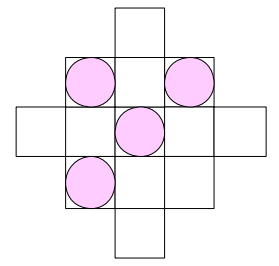

9

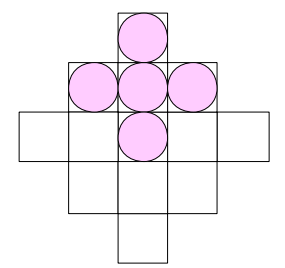

14

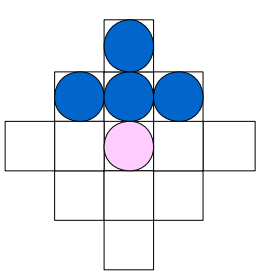

19

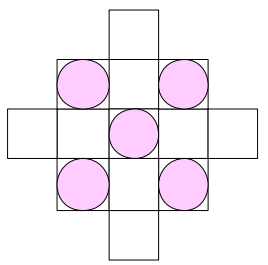

10

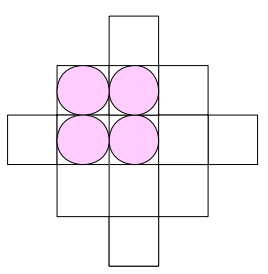

15

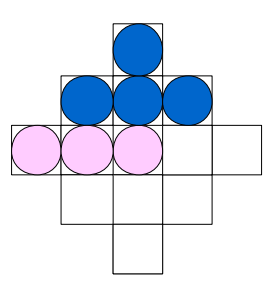

20

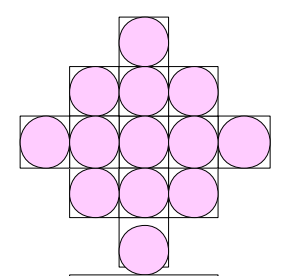

24

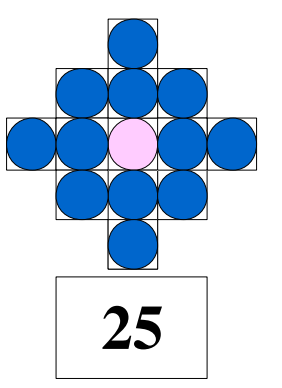



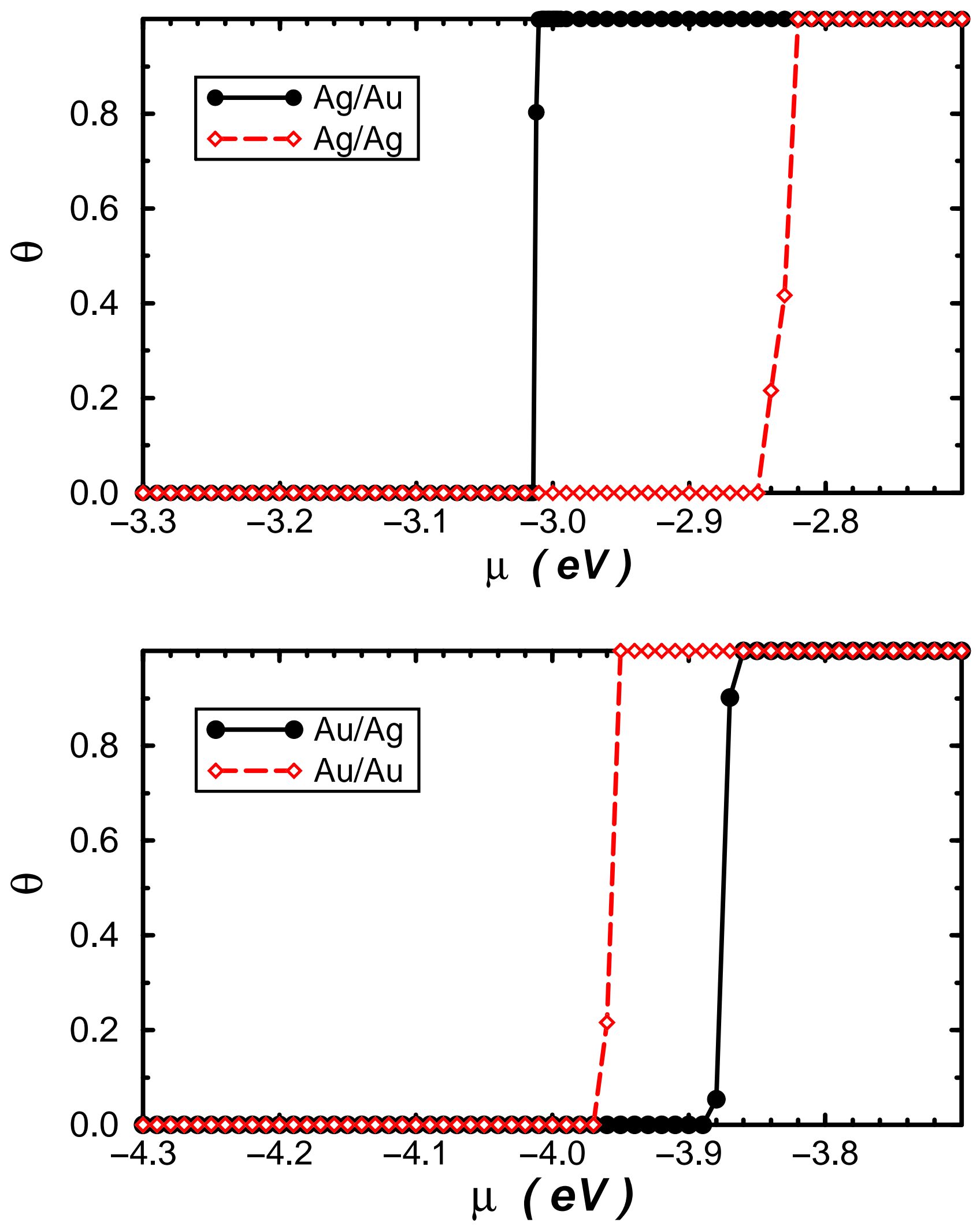
$\mathrm{Ag} / \mathrm{Au}(100)$
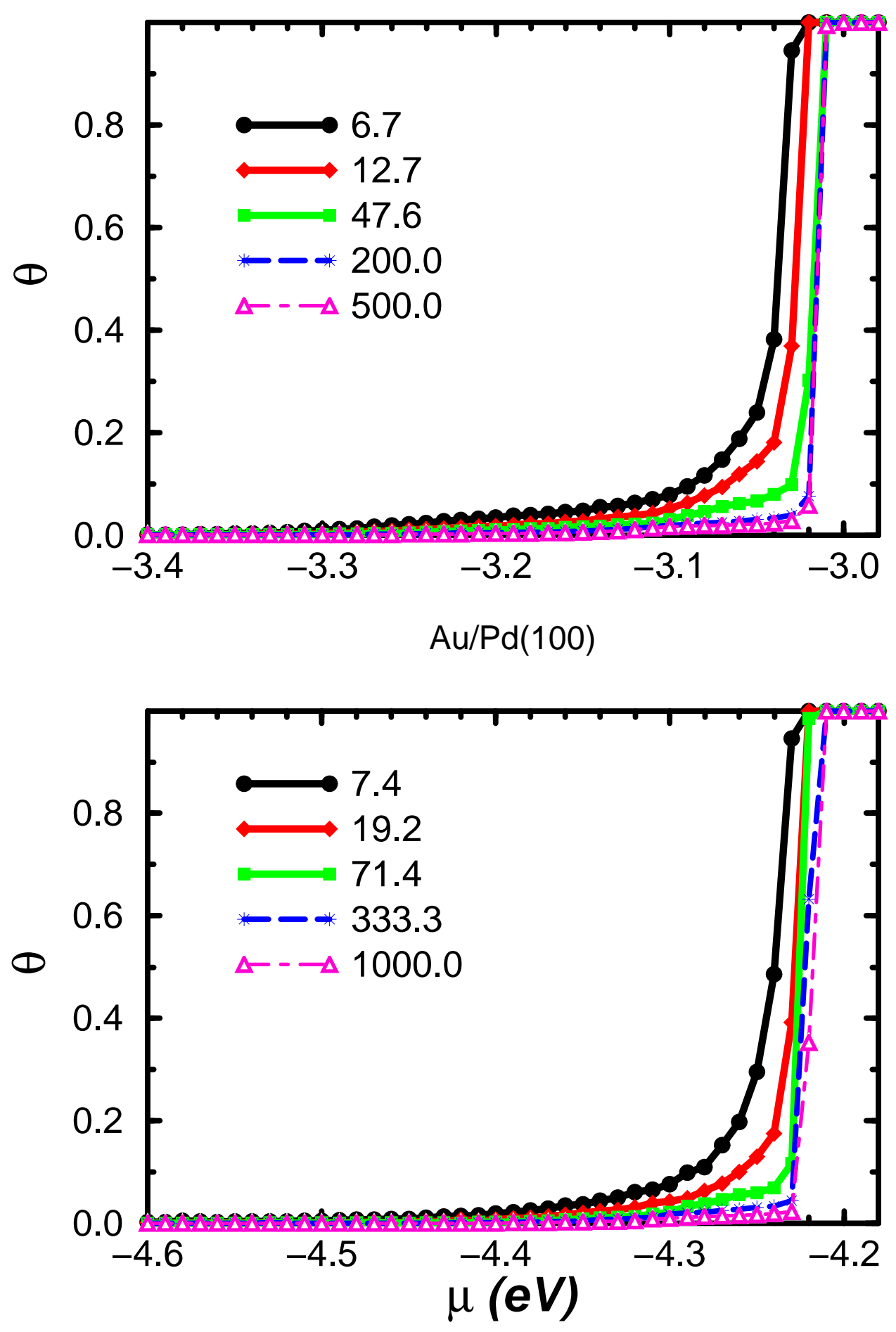

$\mathrm{Ag} / \mathrm{Pt}(100)$
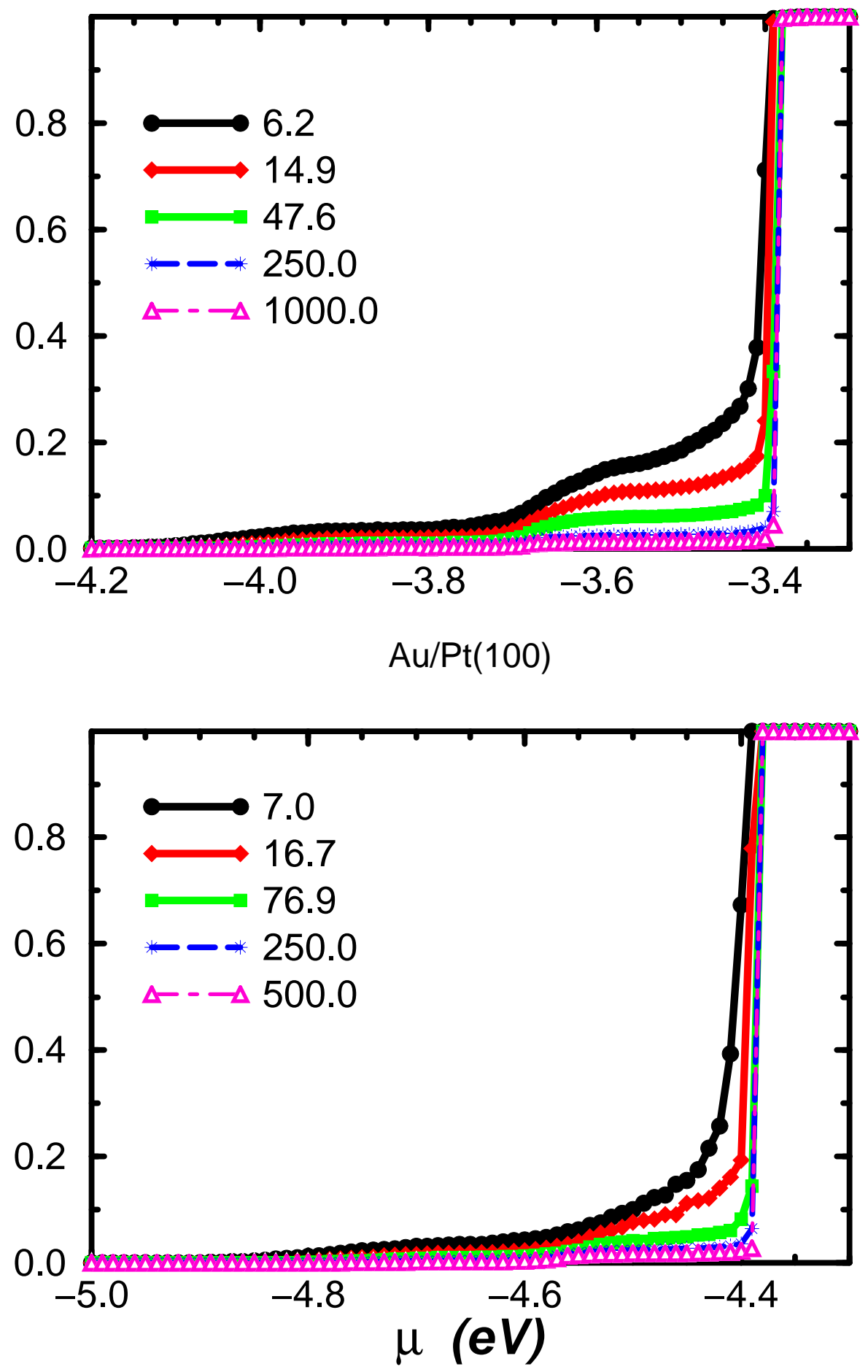

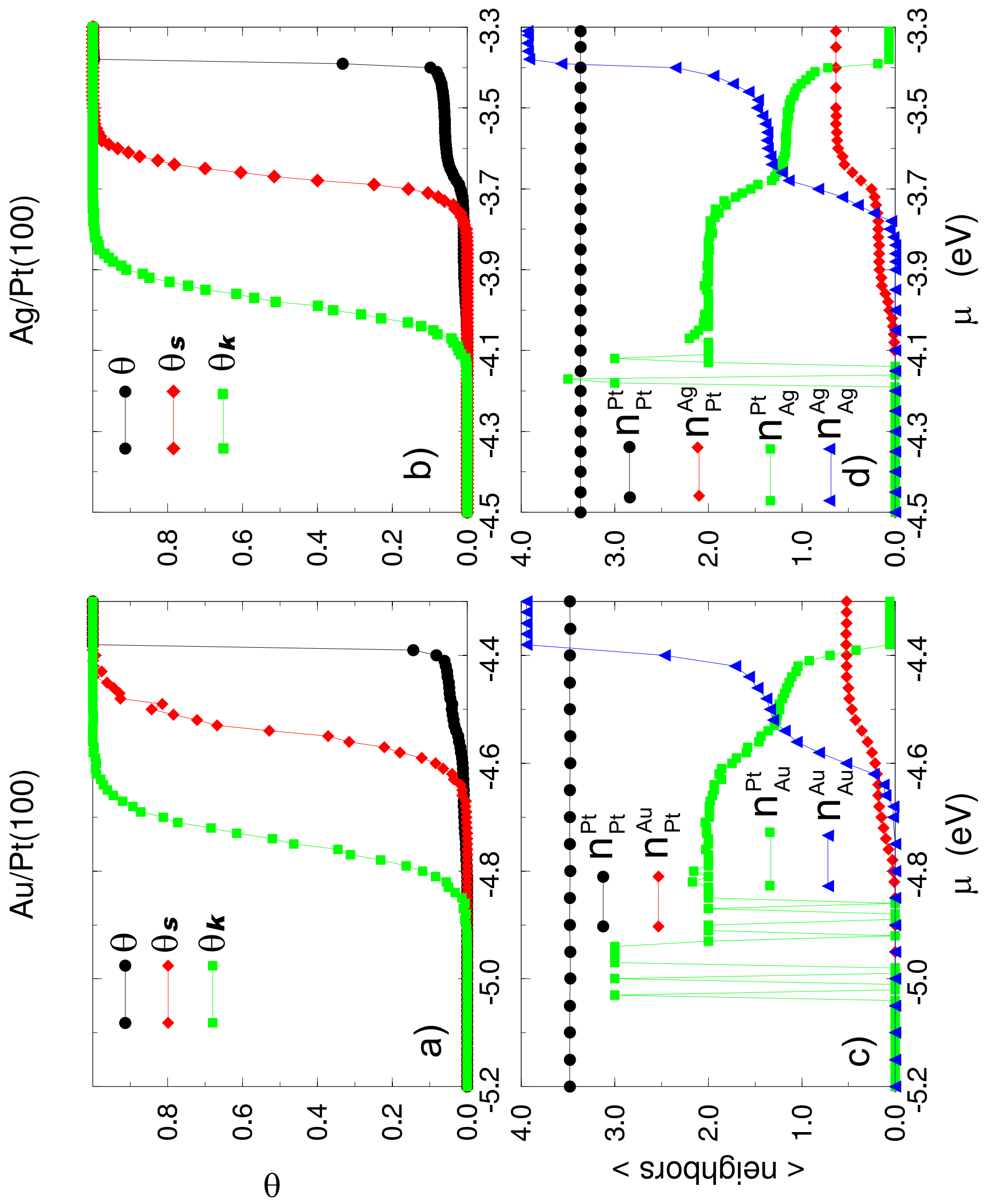

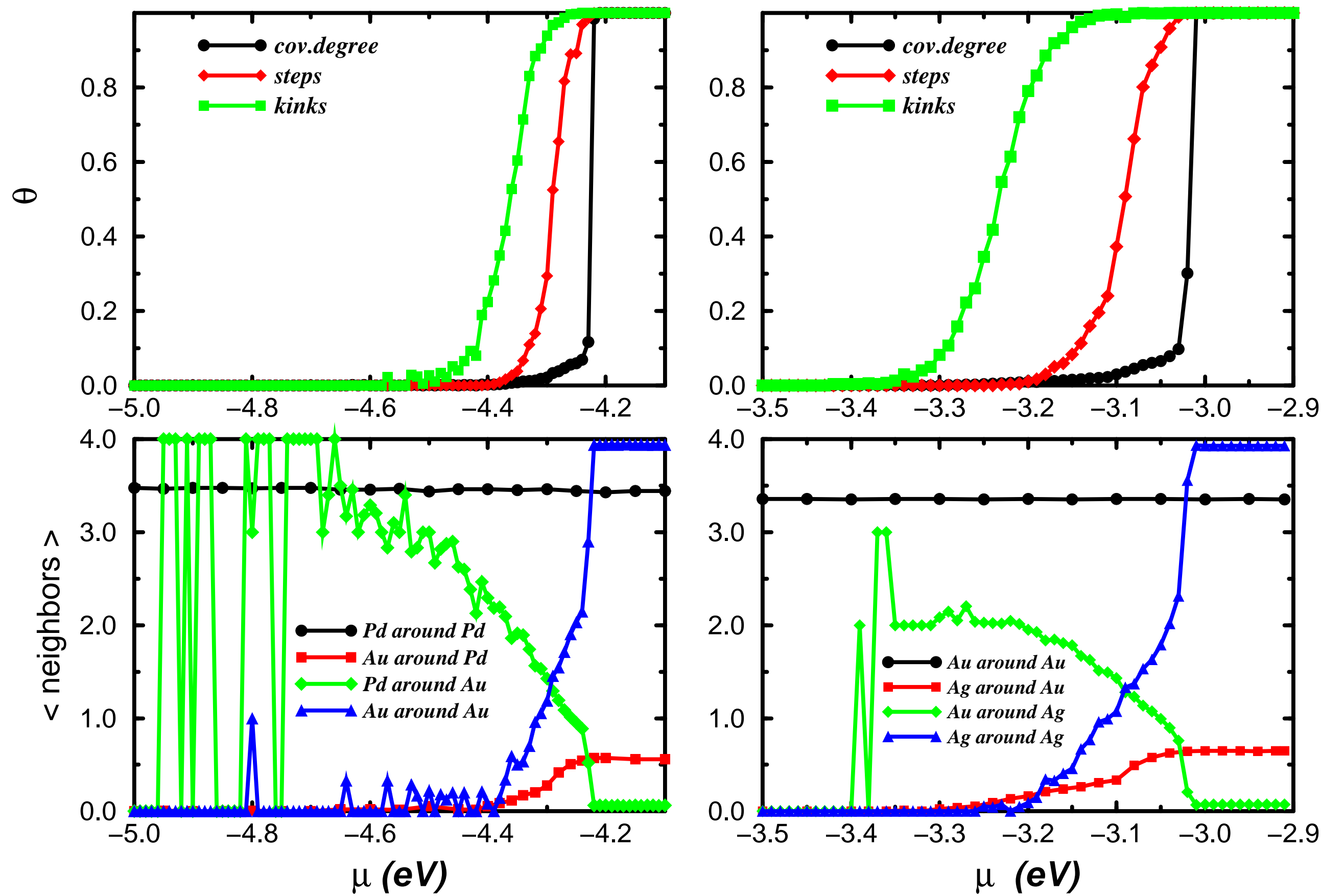
$\mathrm{Au} / \mathrm{Ag}(100)$
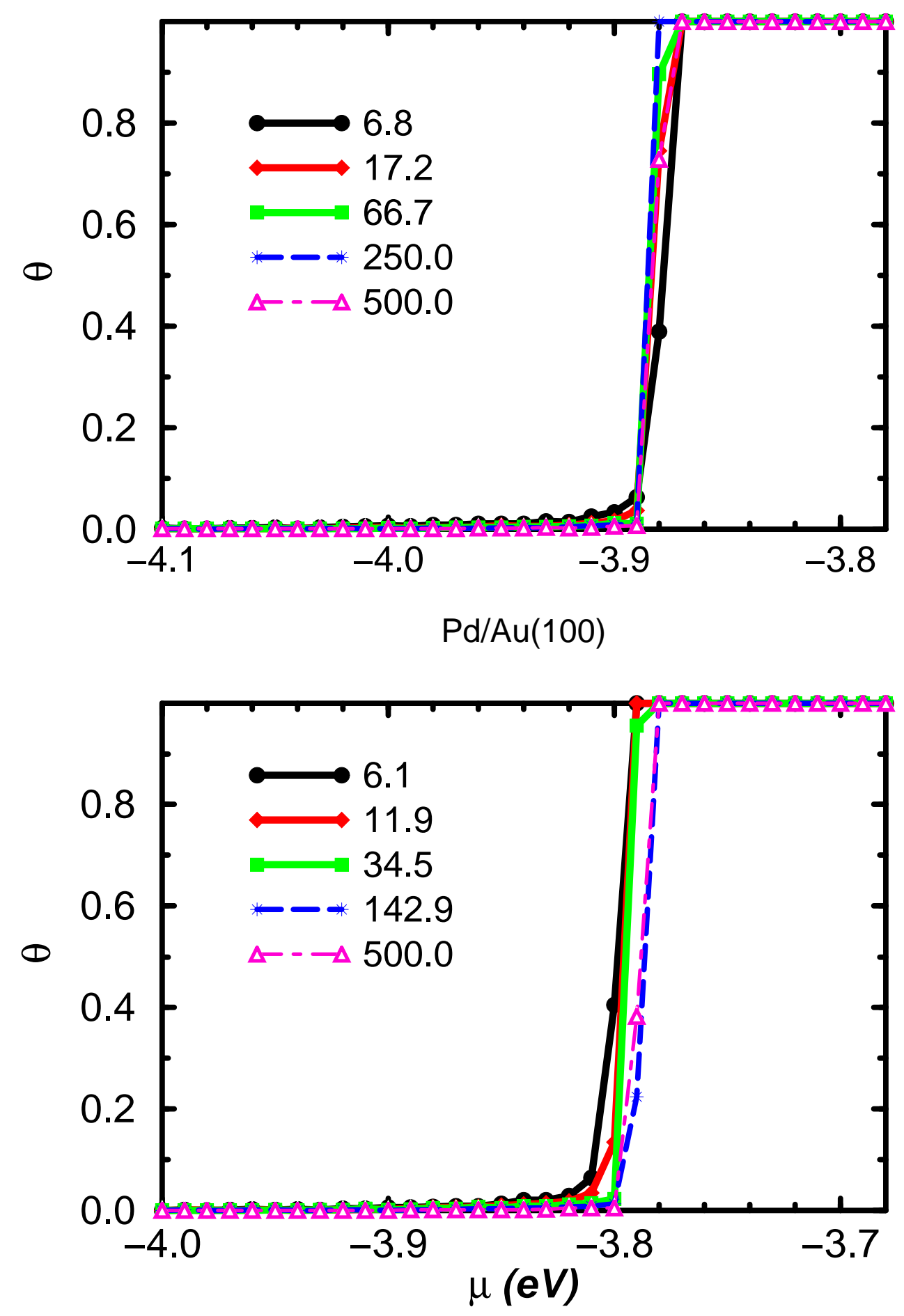

$\mathrm{Pt} / \mathrm{Ag}(100)$
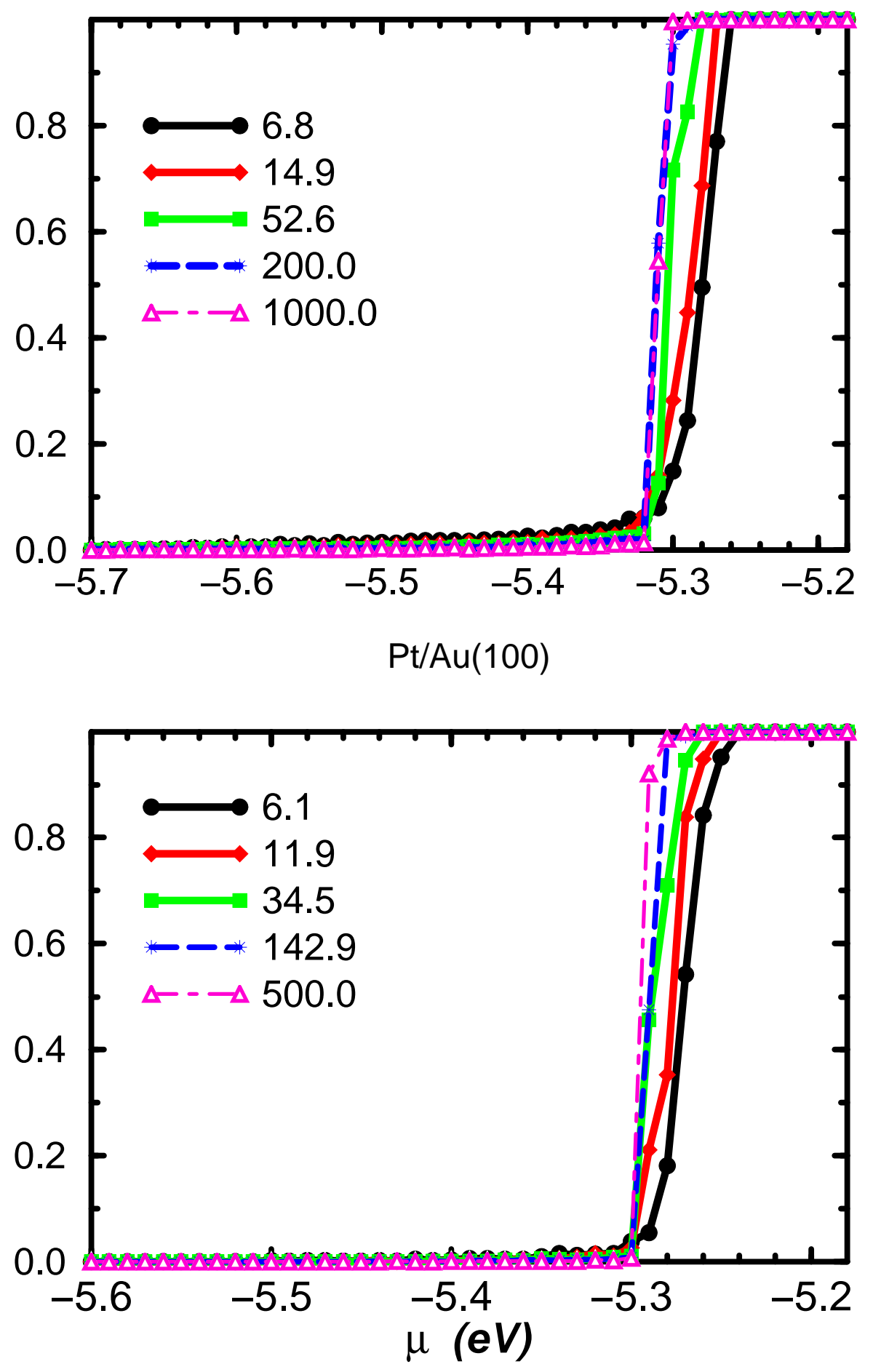

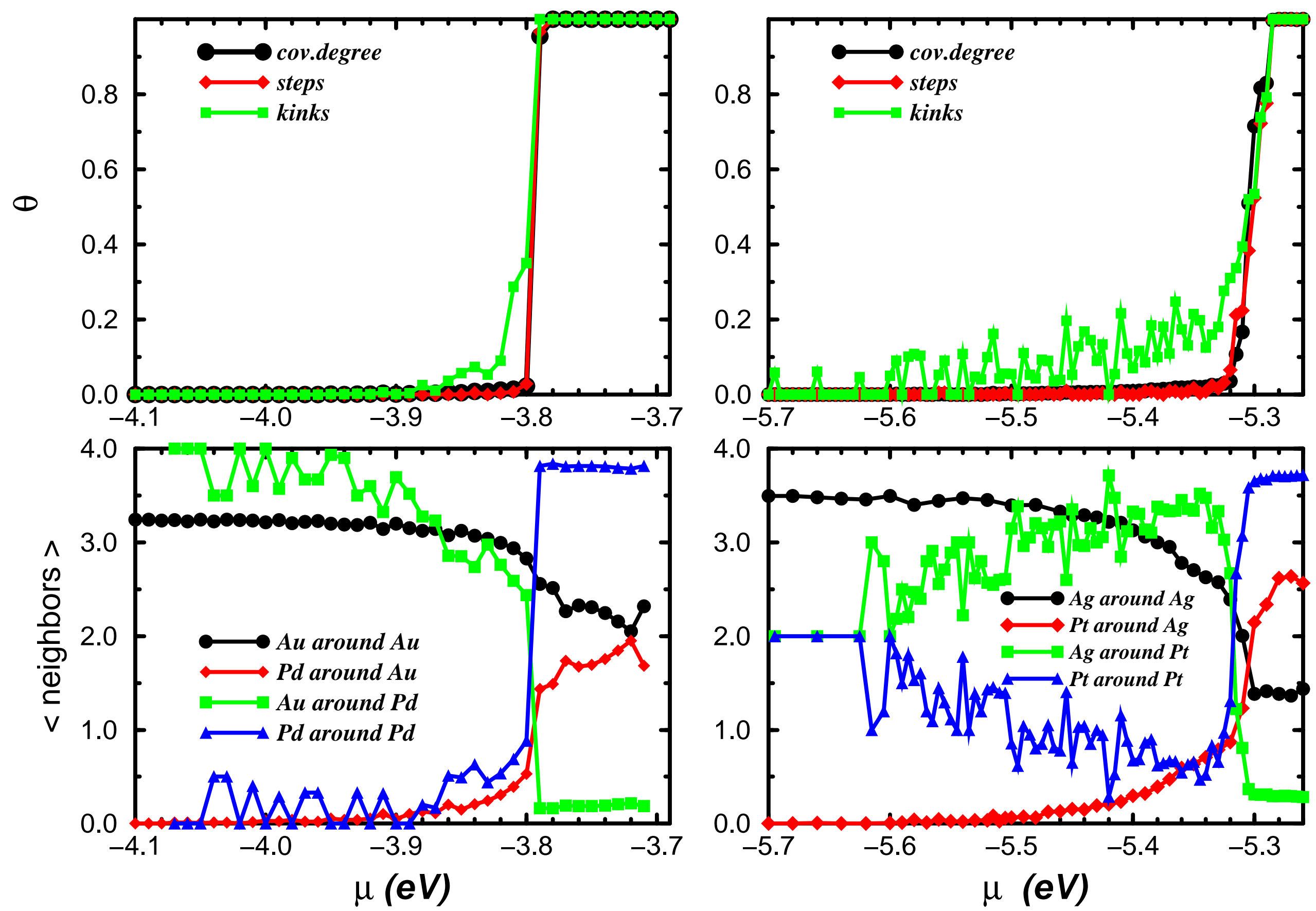

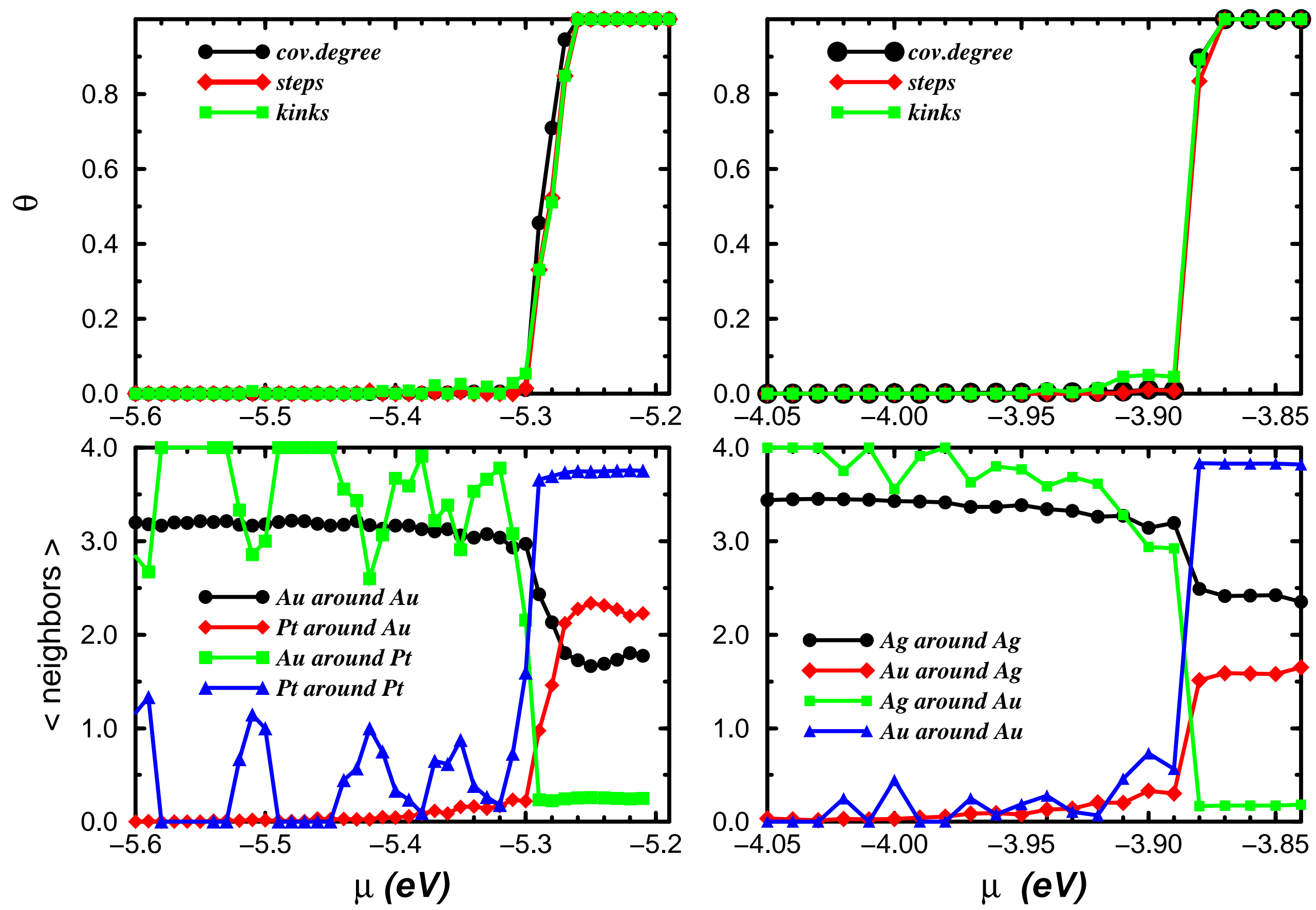


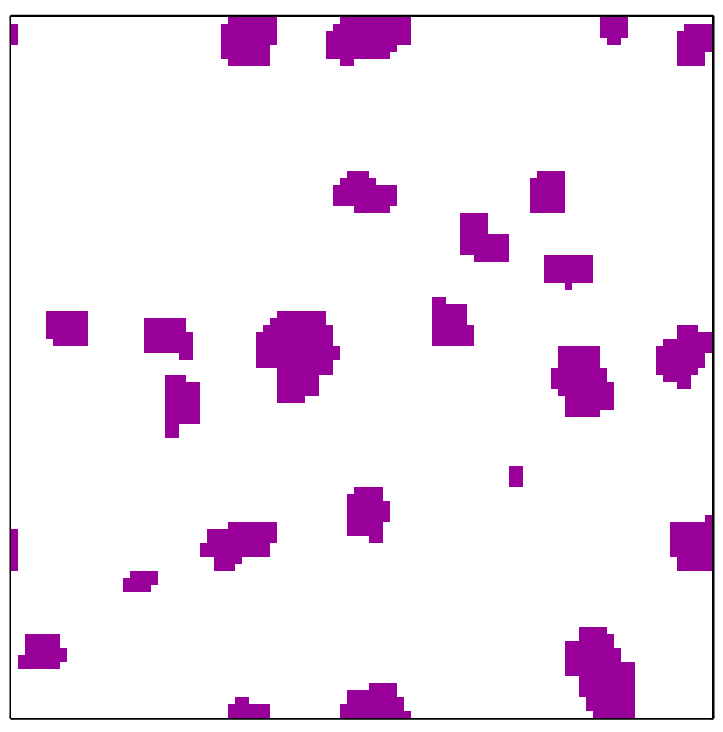

Chom. Pot./oV $=-4.270$

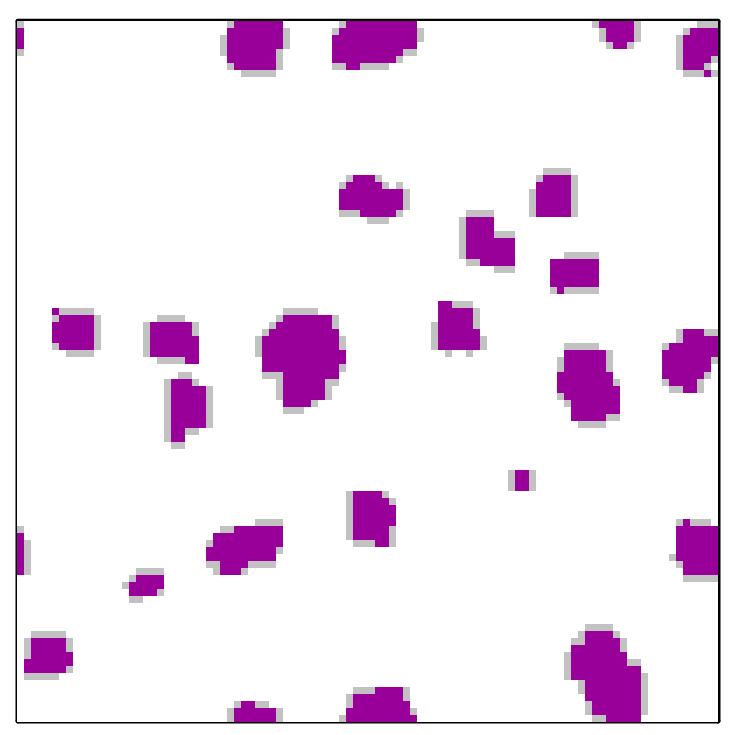

Chem. Pot./oV $=-3.660$

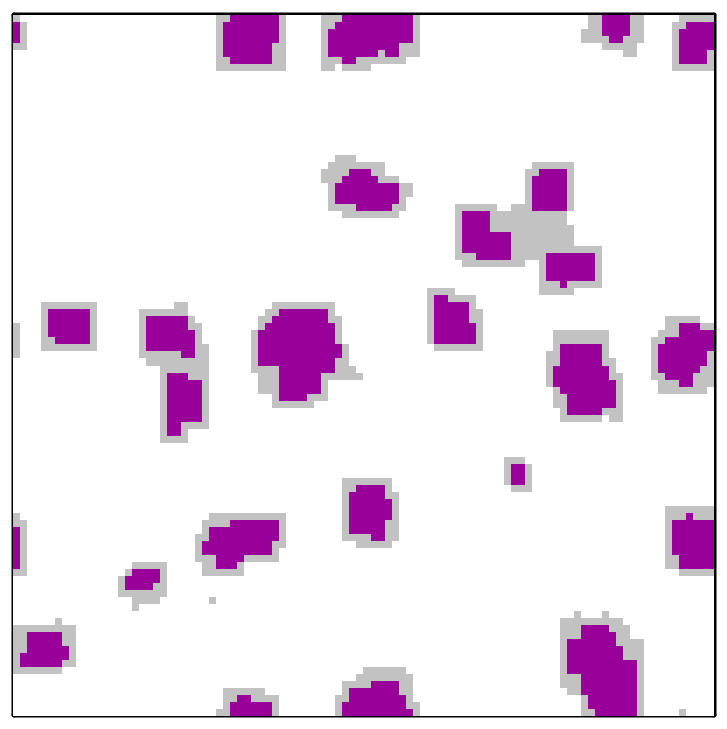

Chom. Pot./ov $=-3.400$

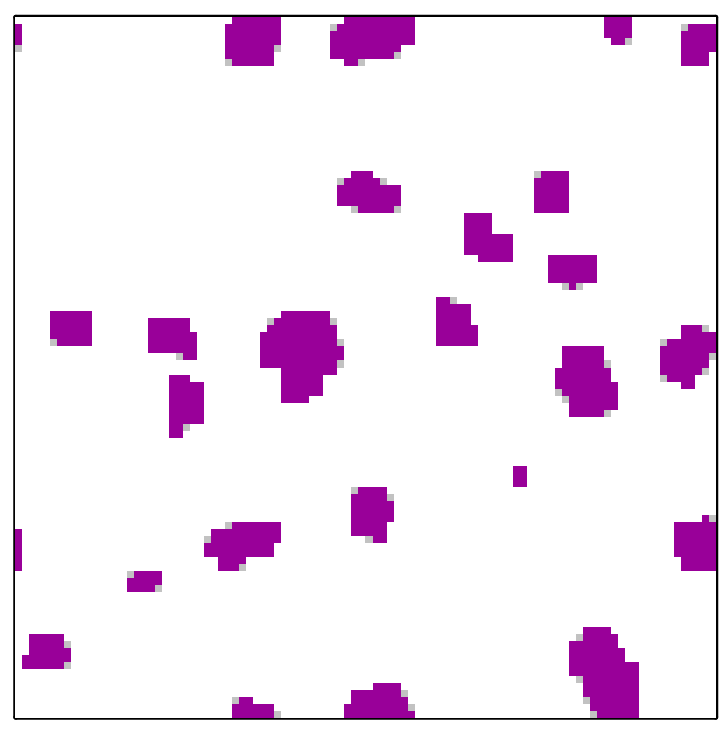

Chem. Pot./ov $=-3.970$

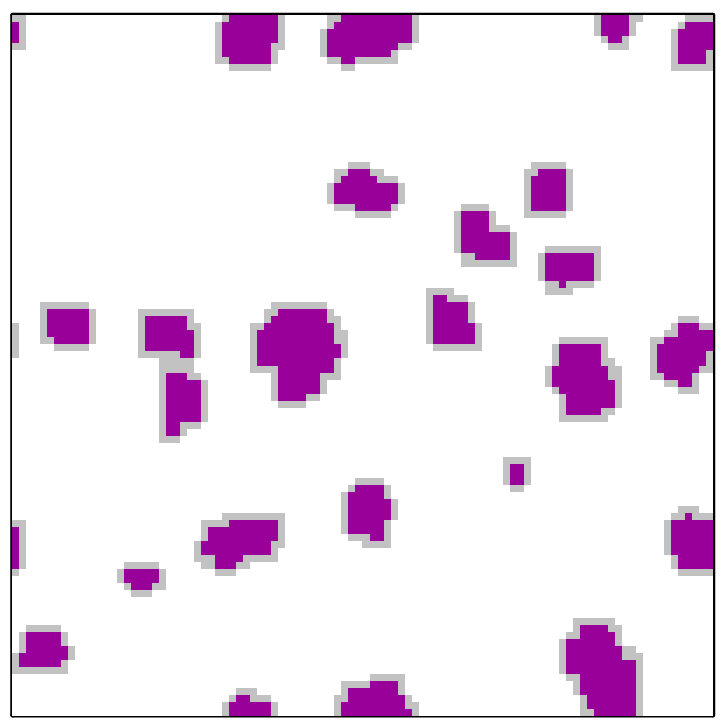

Chem. Pot. $/ \mathrm{OV}=\mathbf{- 3 . 4 4 0}$

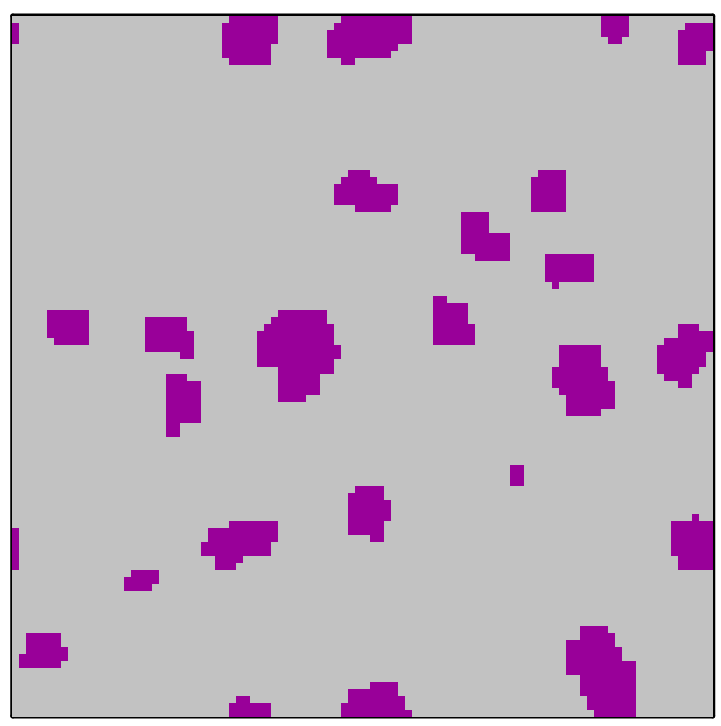

Chem. Pot./oV $=-3.080$ 


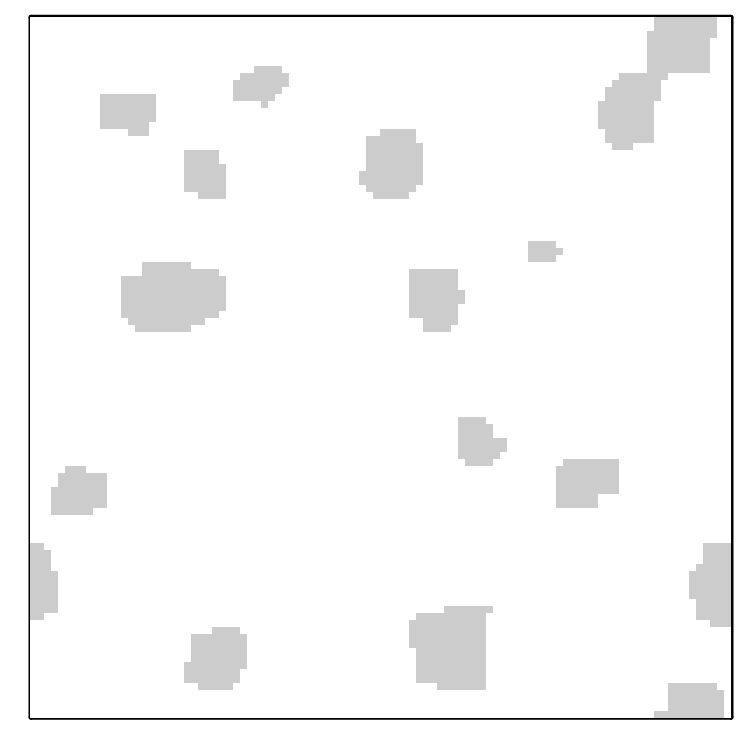

Chem.Pot./ov $=-5.740$

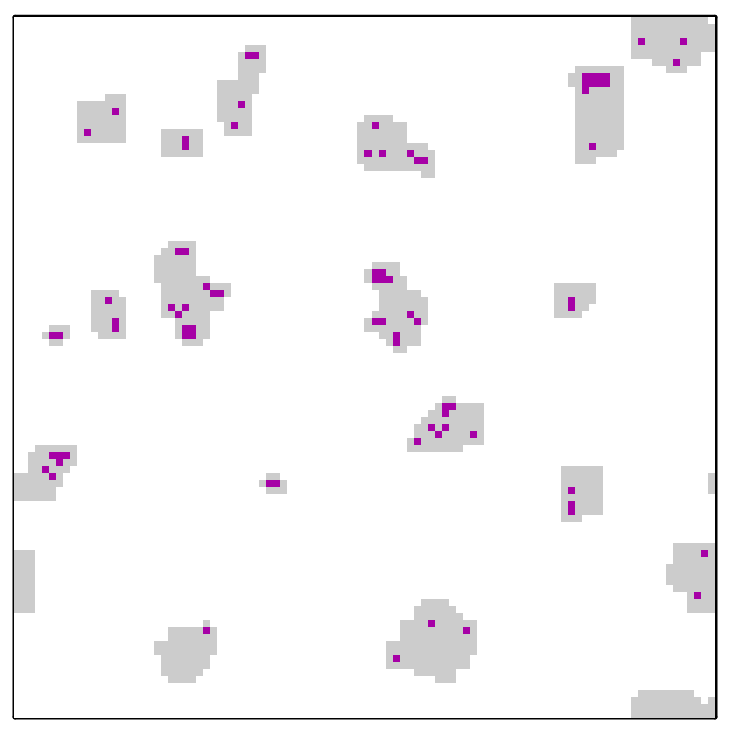

Chem.Pot.ov $=-\mathbf{5 . 4 1 0}$

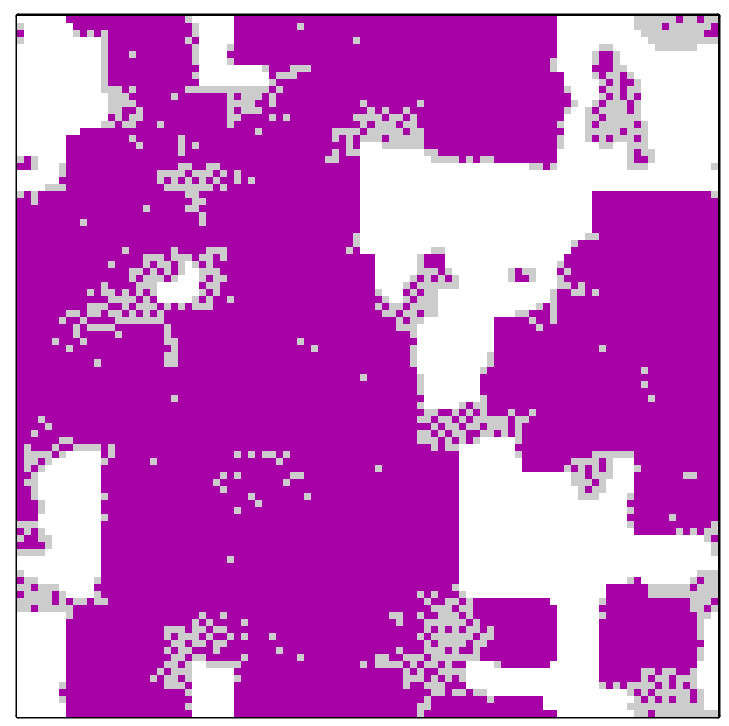

Chem.Pot./oV $=-5.300$

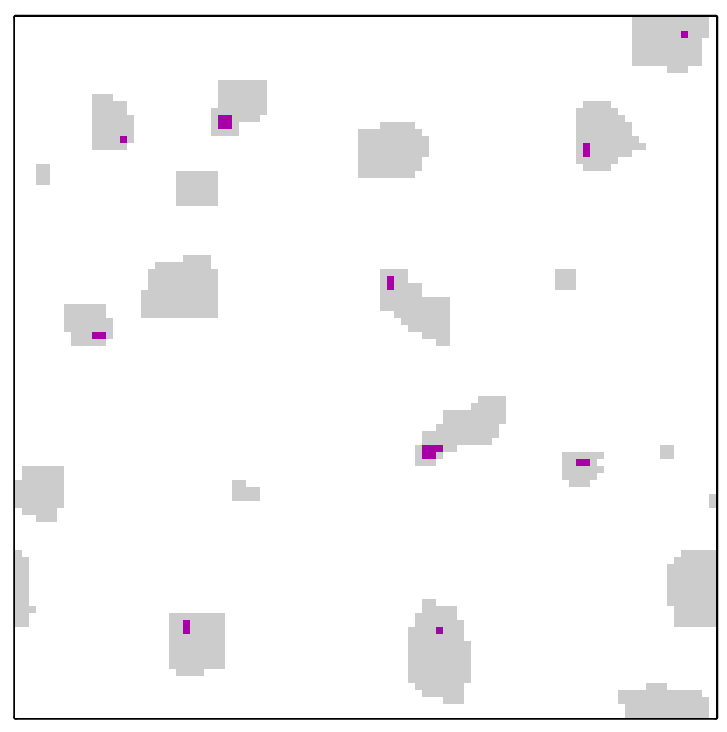

Chem.Pot./ov $=-5.530$

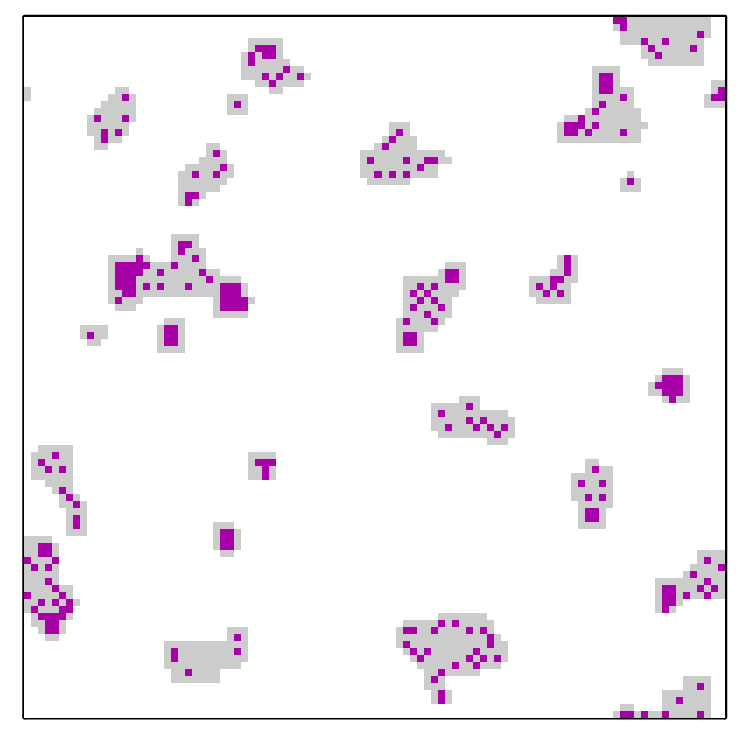

Chem.Pot./ov $=-5.320$

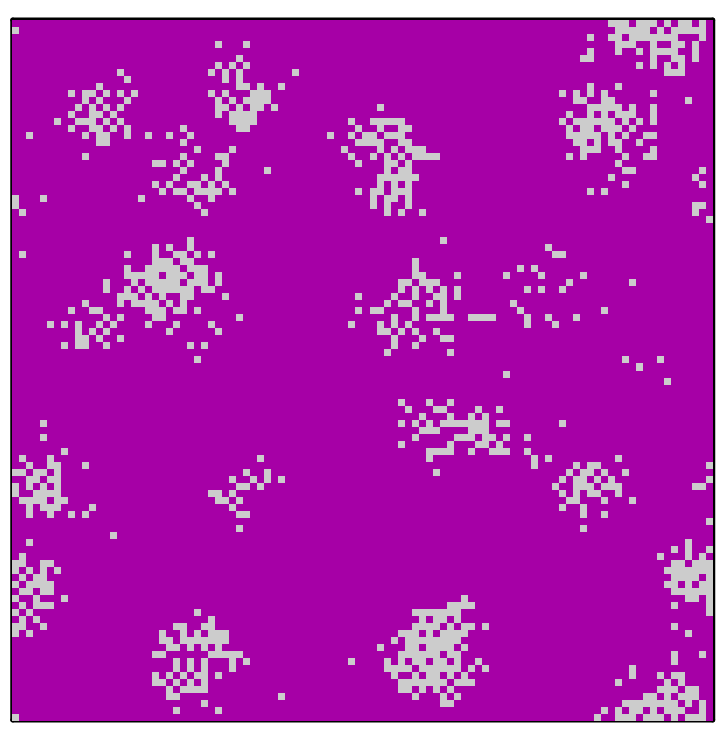

Chom.Pot./ov $=\quad-5.210$ 


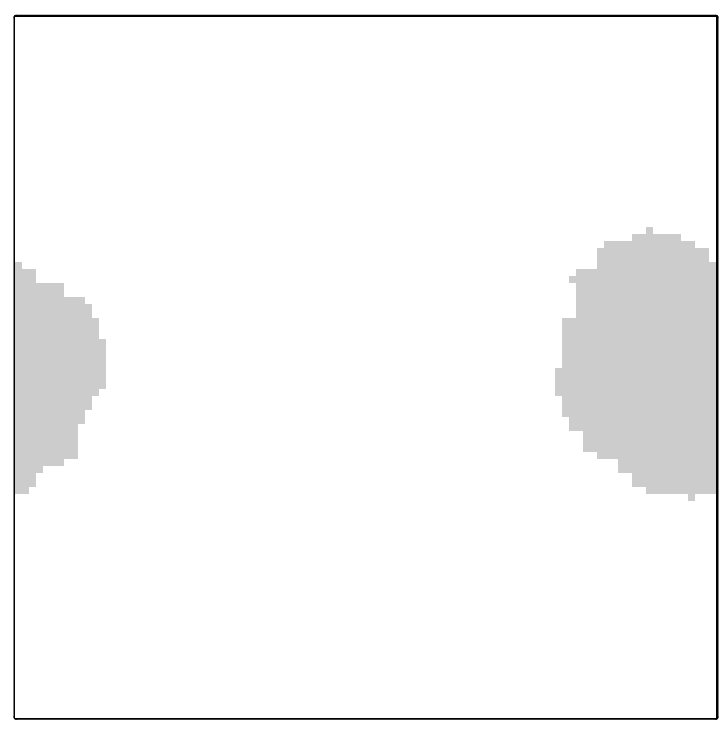

Chem.Pot./ov $=-5.740$

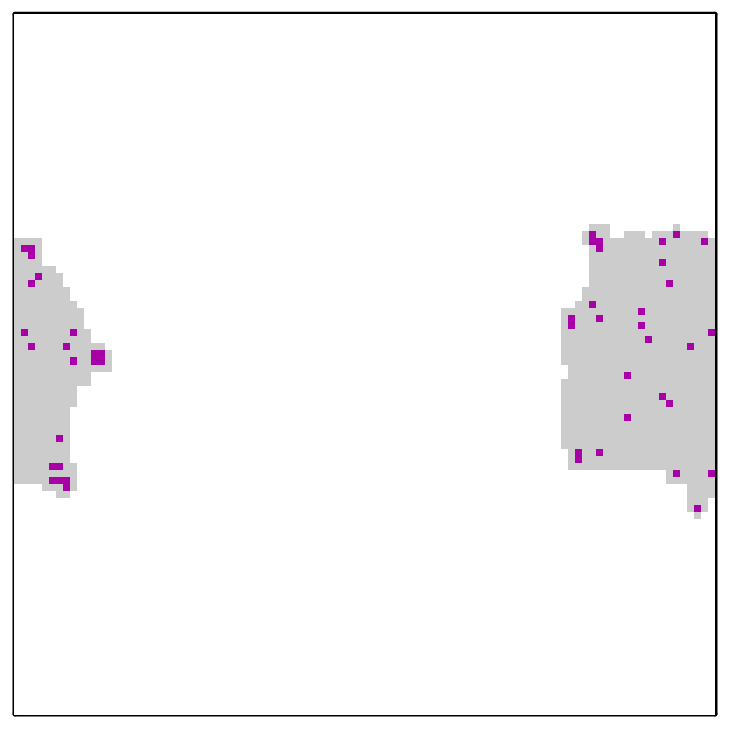

Chem.Pot./ov $=-\mathbf{5 . 4 1 0}$

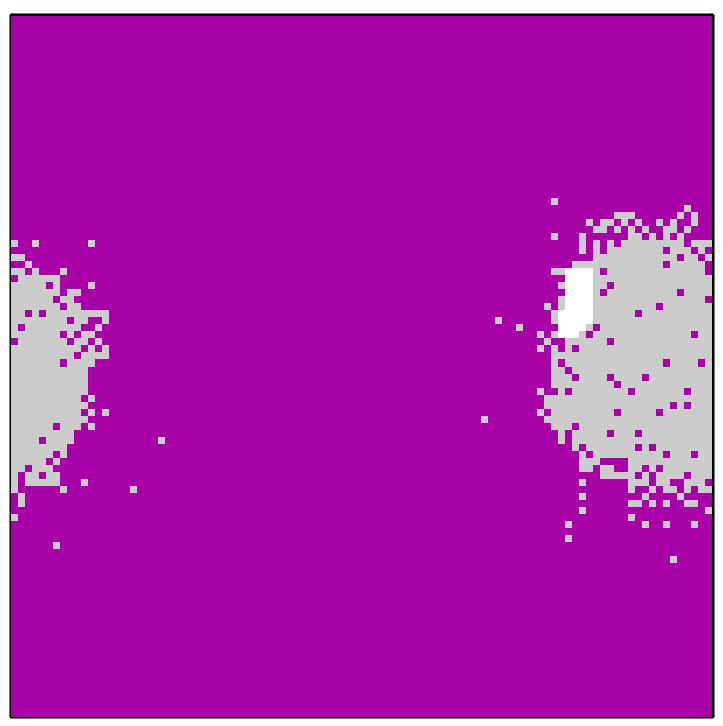

Chem.Pot. $/$ ov $=-5.300$
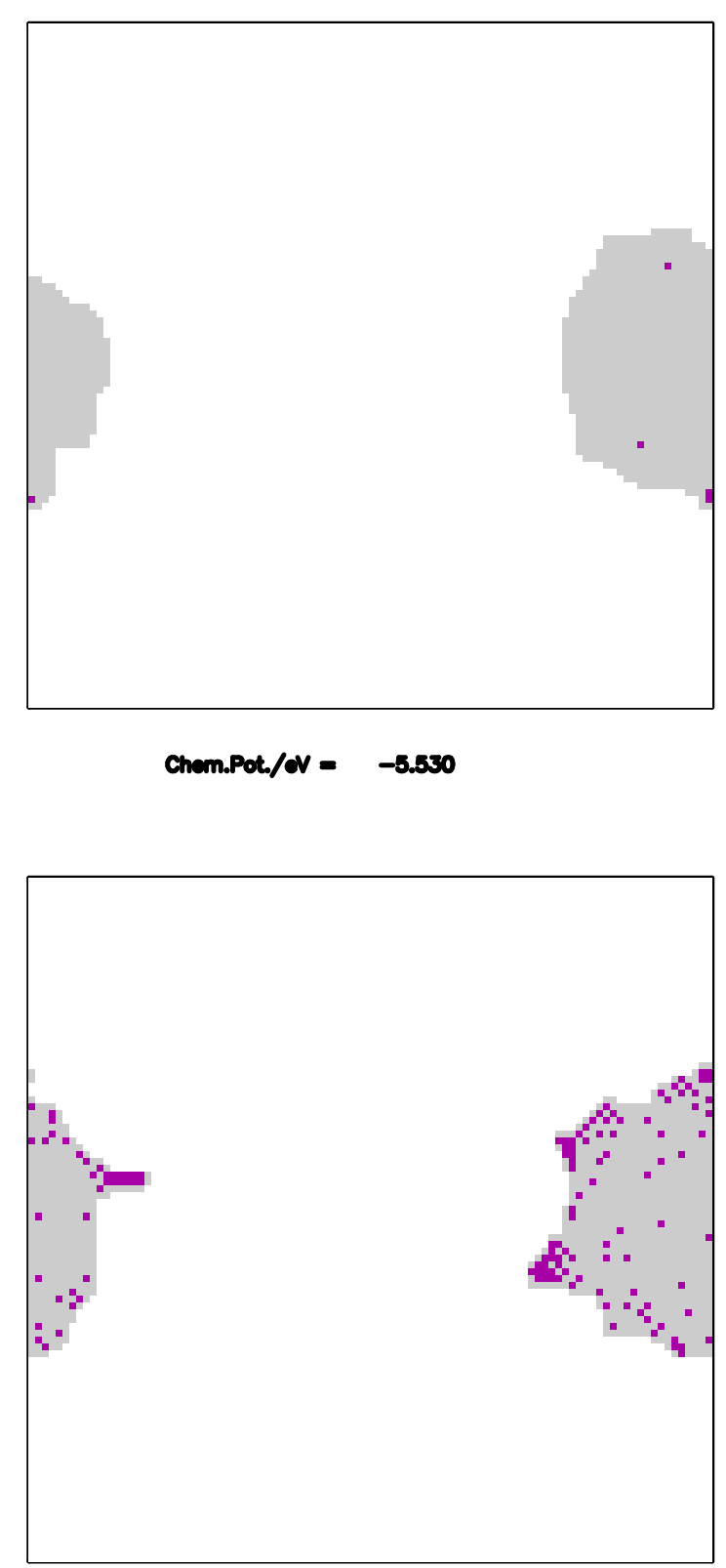

Chem.Pot./ov $=-5.320$

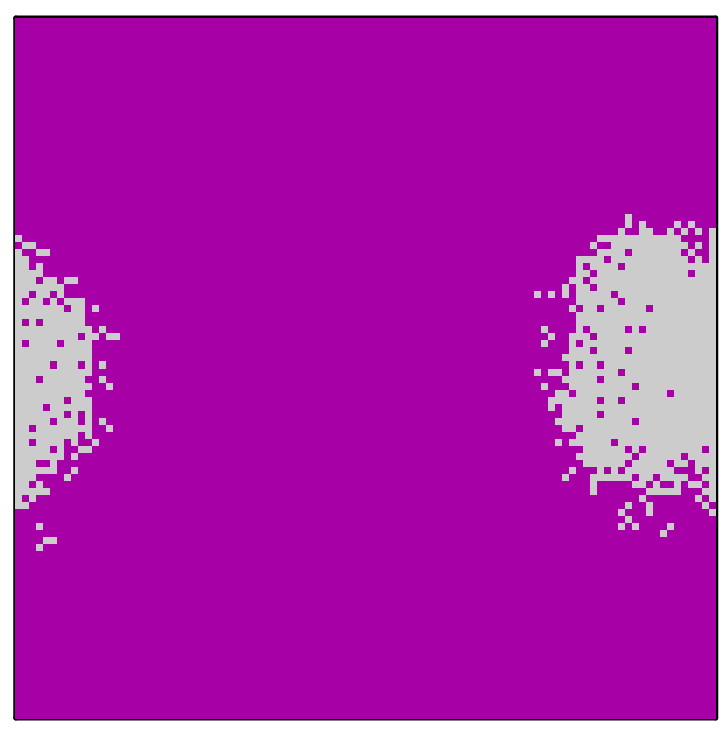

Chem.Pot./ov $=\quad-5.210$ 
$-5.10 \mathrm{eV}$

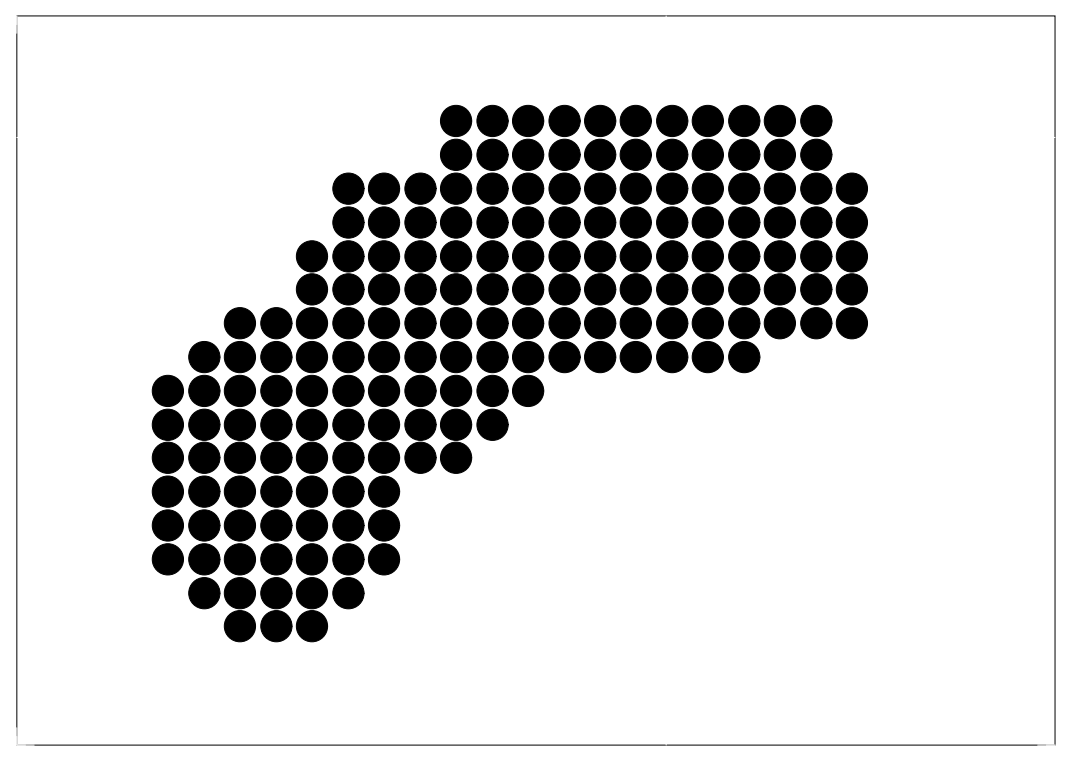

$-4.50 \mathrm{eV}$

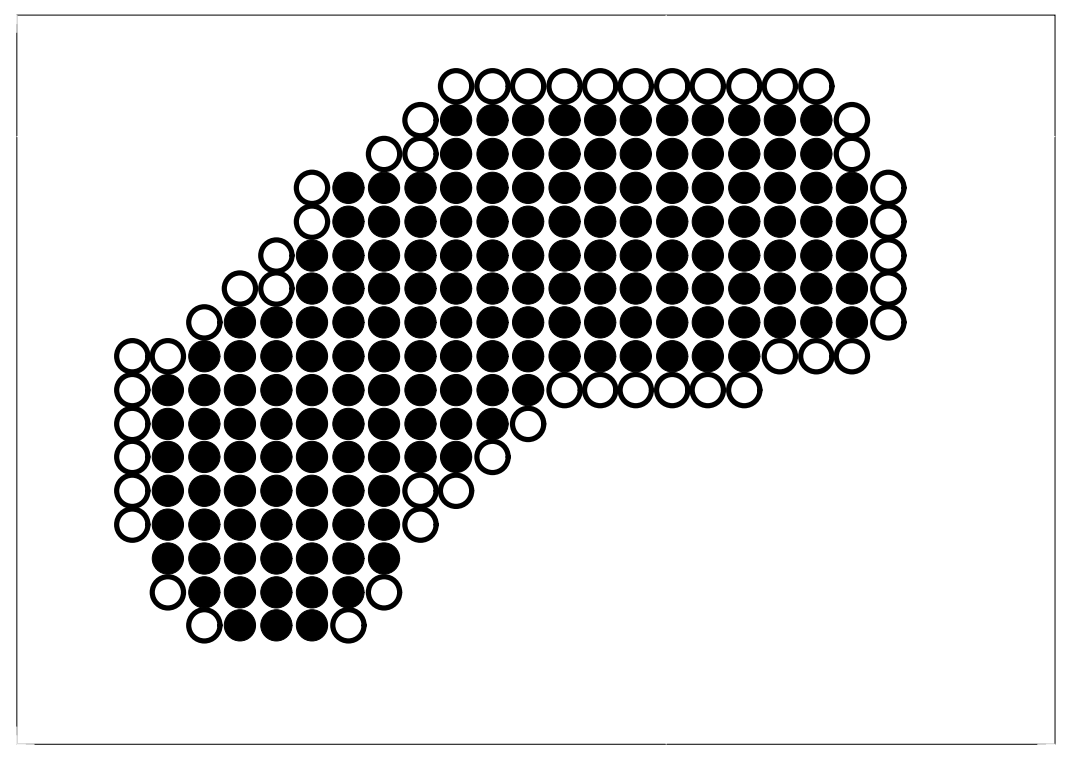

$-4.61 \mathrm{eV}$

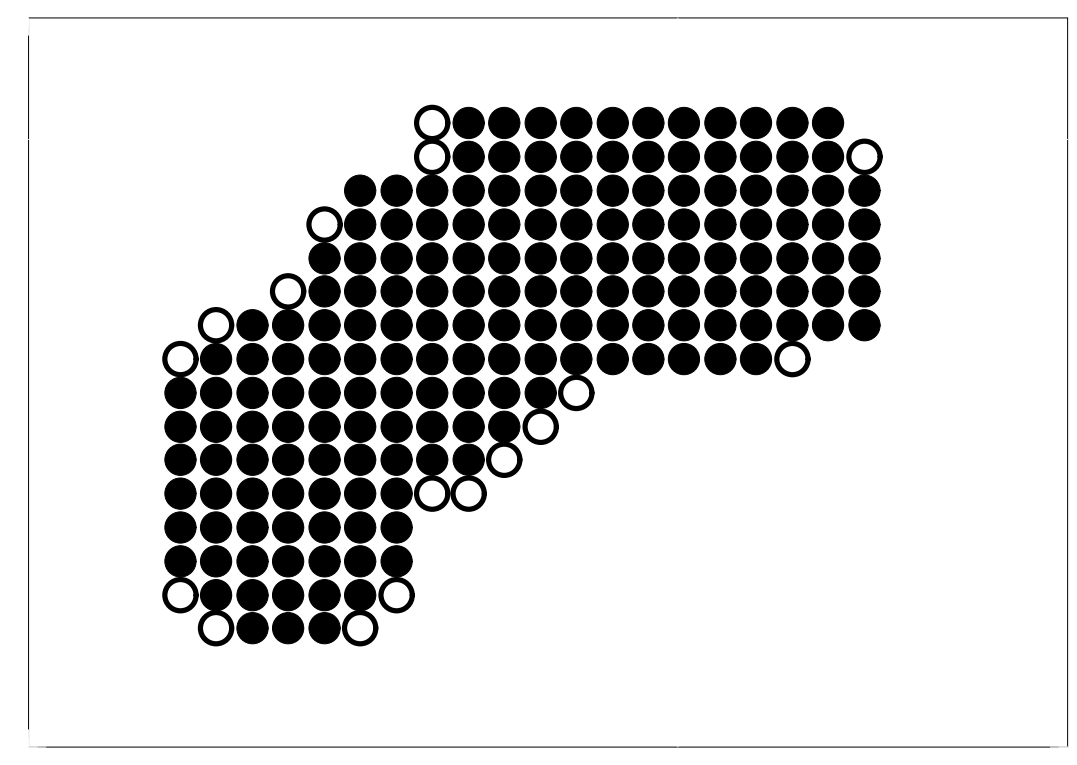

$-4.39 \mathrm{eV}$

89898998989899898980

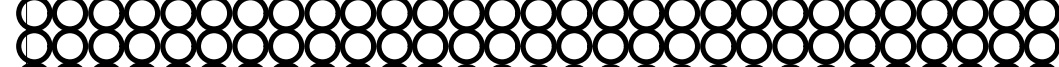

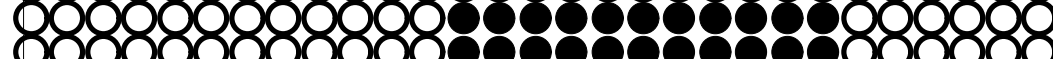

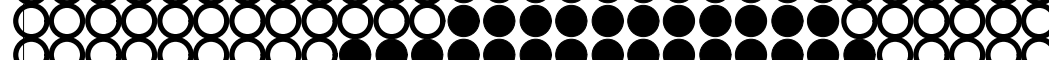
\&b8

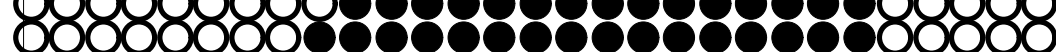
\&8606080 8008060000000008

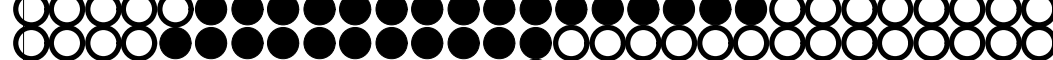
\&808

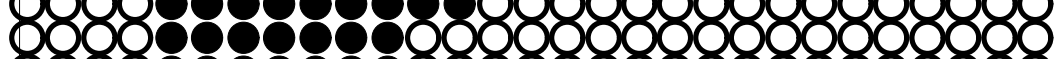
8080000008080808080808 806060 8108018

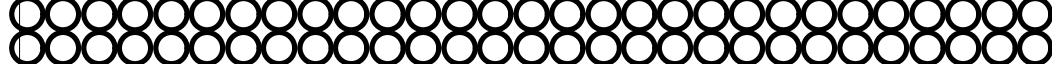
8888080880808880888880888808888 
$-5.65 \mathrm{eV}$

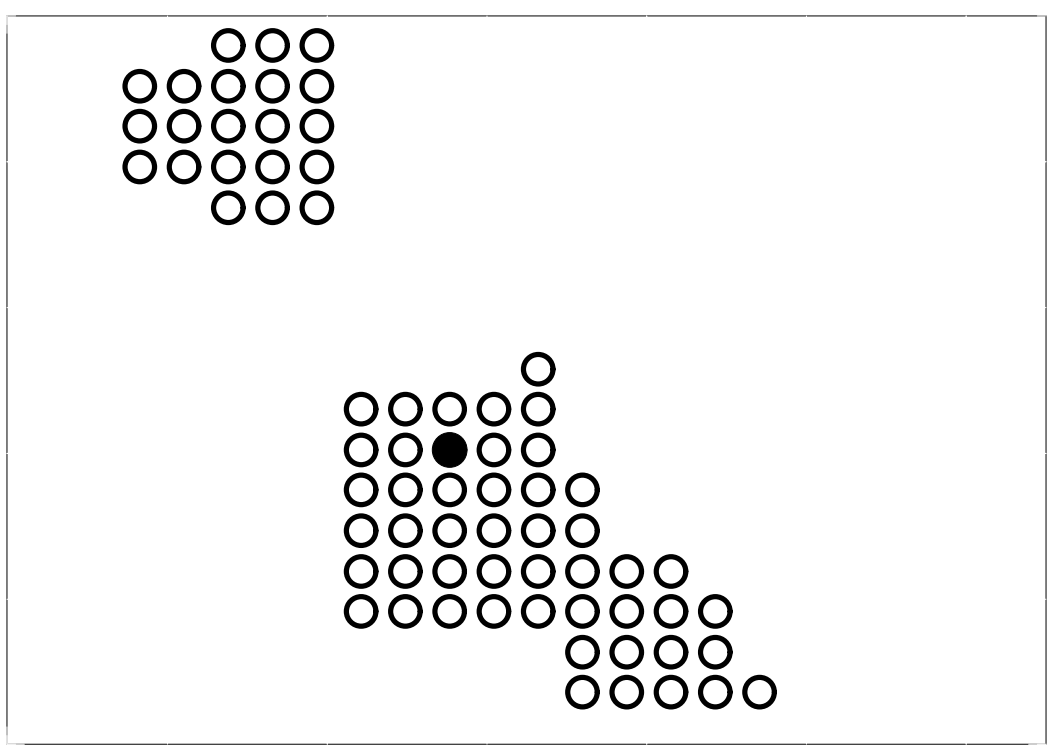

$-5.29 \mathrm{eV}$

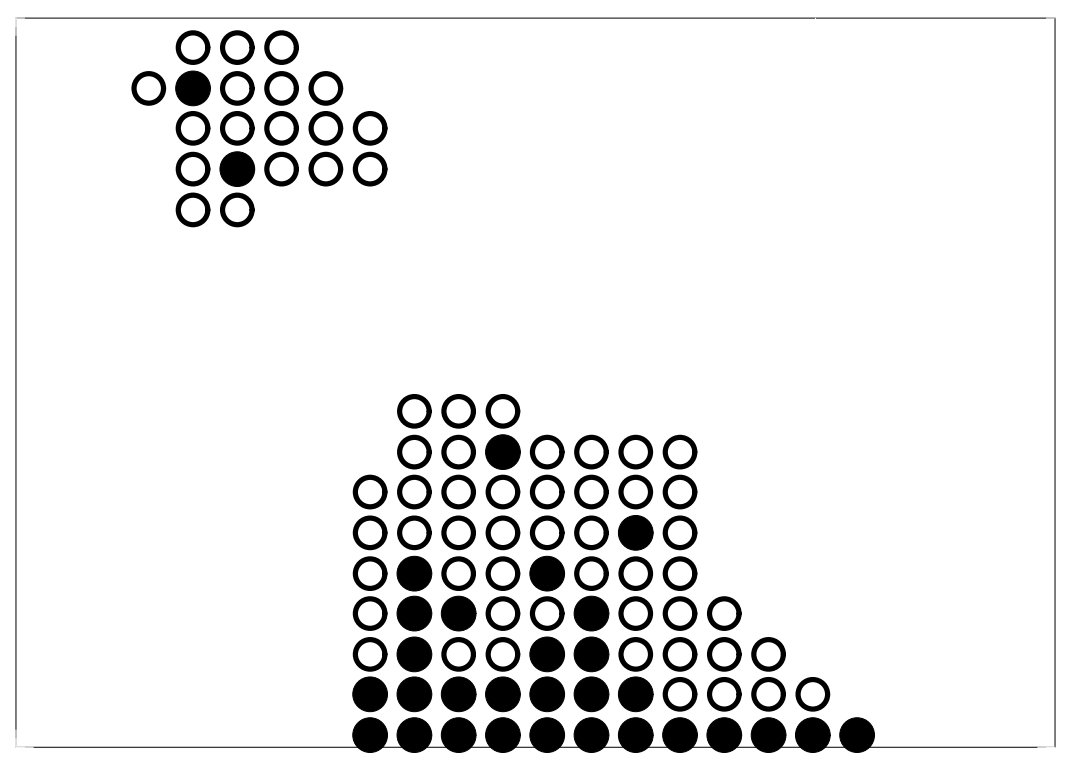

$-5.30 \mathrm{eV}$

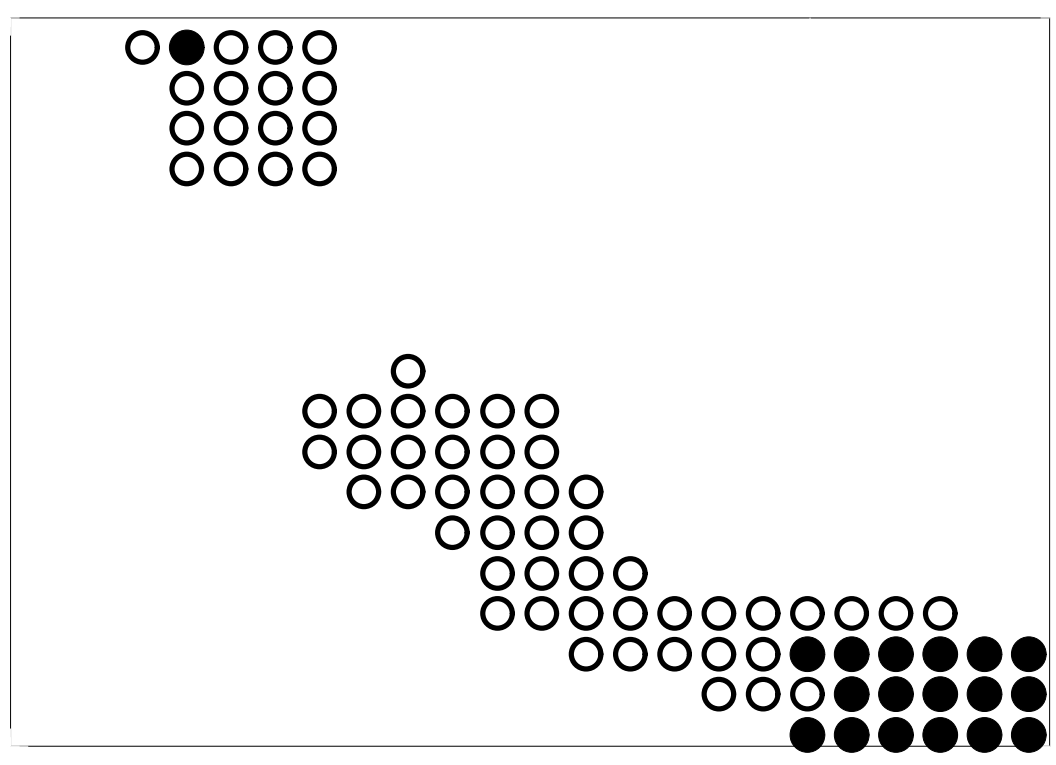

$-5.26 \mathrm{eV}$

99000009999999999999990

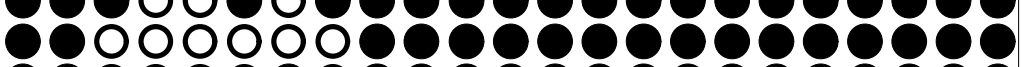

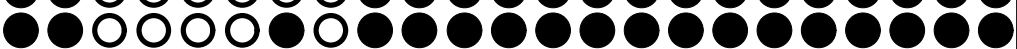

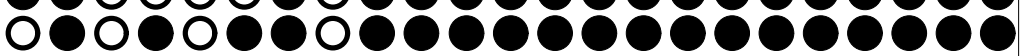

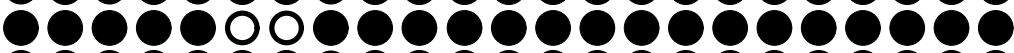

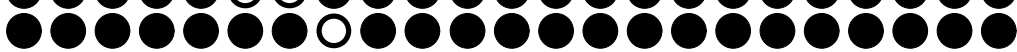

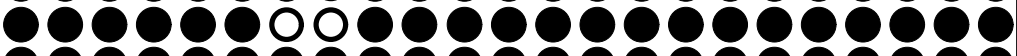

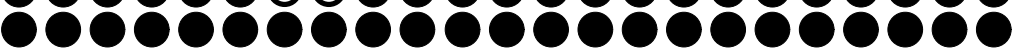

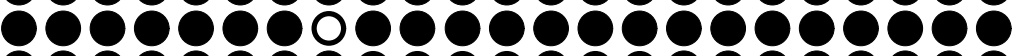

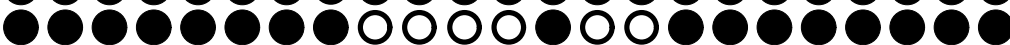

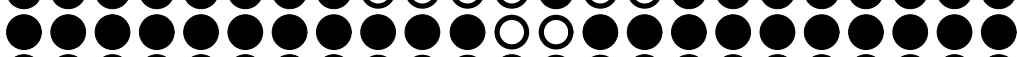

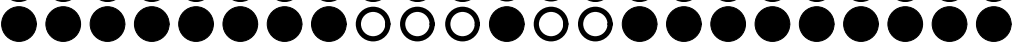

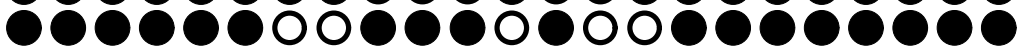

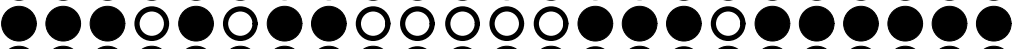

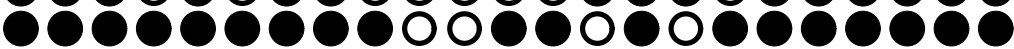

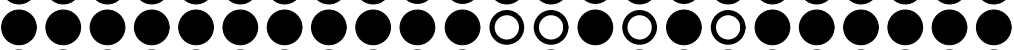
800808098000000080 8688688888008.8008008888 